\title{
La grotte du Sorcier à Saint-Cirq-du-Bugue (Dordogne, France) : nouvelles lectures. Bilan des campagnes 2010 et 2011
}

Romain Pigeaud, Florian Berrouet, Estelle Bougard, Hervé Paitier, Vincent Pommier et Pascal Bonic

\section{(2) OpenEdition}

\section{Journals}

Édition électronique

URL : http://journals.openedition.org/paleo/2455

DOI : $10.4000 /$ paleo.2455

ISSN : 2101-0420

Éditeur

SAMRA

\section{Édition imprimée}

Date de publication : 15 décembre 2012

Pagination : 223-248

ISSN : $1145-3370$

\section{Référence électronique}

Romain Pigeaud, Florian Berrouet, Estelle Bougard, Hervé Paitier, Vincent Pommier et Pascal Bonic, « La grotte du Sorcier à Saint-Cirq-du-Bugue (Dordogne, France) : nouvelles lectures. Bilan des campagnes 2010 et 2011 », PALEO [En ligne], 23 | 2012, mis en ligne le 19 avril 2013, consulté le 25 juillet 2020. URL : http://journals.openedition.org/paleo/2455; DOI : https://doi.org/10.4000/paleo. 2455

Ce document a été généré automatiquement le 25 juillet 2020.

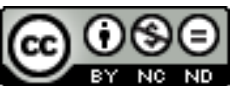

PALEO est mis à disposition selon les termes de la licence Creative Commons Attribution - Pas d'Utilisation Commerciale - Pas de Modification 4.0 International. 


\section{La grotte du Sorcier à Saint-Cirq- du-Bugue (Dordogne, France) : nouvelles lectures. Bilan des campagnes 2010 et 2011}

Romain Pigeaud, Florian Berrouet, Estelle Bougard, Hervé Paitier, Vincent Pommier et Pascal Bonic

\section{NOTE DE L'ÉDITEUR}

Avec la collaboration de Marie-Dominique PINEL, Marie-Laure LATREILLE et Alice REDOU.

Cet article est dédié à la mémoire de Norbert AUJOULAT.

\section{Introduction}

La grotte du Sorcier, sur la commune de Saint-Cirq-du-Bugue, se situe à quelques kilomètres en aval du village des Eyzies en direction du Bugue, peu avant la dernière grotte proche de la Vézère qu'est Bara-Bahau (Delluc et Delluc 2009). Cette cavité partiellement comblée, dont l'entrée a servi d'habitat troglodytique comme nombre de cavités et autres cluzeaux que l'on rencontre en Périgord, s'ouvre au sud-est dans un calcaire coniacien (Aujoulat 2006). Elle se présente comme un rectangle d'environ 5 à 6 $\mathrm{m}$ de large pour une longueur de $13 \mathrm{~m}$. La partie profonde de la grotte est marquée par un abaissement $\mathrm{du}$ sol rocheux de plus d'un mètre, témoignant d'un aménagement partiel des lieux à des fins touristiques - la «tranchée » encore en place permet de contempler à distance suffisante les gravures de la voûte. Le plafond est parcouru dans le sens de pénétration de la cavité par une diaclase dont la largeur varie entre $0,50 \mathrm{~m}$ et 
$1,50 \mathrm{~m}$ et qui a certainement joué un rôle, d'un point de vue topographique, dans l'ornementation de la grotte (Delluc et Delluc 1984).

2 Les représentations préhistoriques ont été découvertes le 22 mai 1952 par Noël Brousse, alors propriétaire des lieux et cousin du préhistorien Séverin Blanc. Le classement aux Monuments historiques interviendra le 19 novembre 1958. Plusieurs études vont se succéder : une étude préliminaire d'André Glory qui fera l'objet d'une publication partielle (Blanc 1955); une série de relevés par L. Dams (1980), qui ont le mérite de proposer une vision à peu près exhaustive des traits gravés et des accidents de paroi mais qui doivent être relativisés ${ }^{1}$; enfin, une étude scientifique et documentée menée par B. et G. Delluc (Delluc et al. 1987) conduira à la publication de la première monographie consacrée à la grotte, sous forme d'article. Mentionnons en outre une étude climatologique réalisée dans les années 1970 par J. Brunet et P. Vidal (1978). En 1966, des moulages, réalisés les 12 et 13 avril par l'équipe d'André Glory, ont fortement endommagé les parois et les ont rendues, pour certaines, imperméables (Delluc et al. 1987 - p. 380). La grotte du Sorcier est privée et ouverte au public depuis les années $1970^{2}$.

\section{Une nouvelle campagne d'étude de la grotte du Sorcier}

En 2009, l'un de nous (RP) avait été sollicité par l'actuel propriétaire, Jean-Max Touron, pour établir un inventaire des représentations gravées et de l'état des parois de la grotte, peu profonde et dont les secteurs ornés, assez proches de l'extérieur, restent à la merci de divers types d'altérations. Nous avions alors mis en évidence un grignotage de la paroi par des altéragènes (Aujoulat 2006) ainsi que plusieurs actes de vandalisme ${ }^{3}$ qui avaient porté atteinte à l'intégrité des figures (voir infra). La problématique des missions 2010 et 2011 était donc, tout d'abord, de faire le point sur l'état de conservation des gravures et les éventuelles menaces auxquelles elles étaient exposées. Ensuite, à partir du déchiffrement minutieux des tracés qu'avaient réalisé B. et G. Delluc, il nous a semblé judicieux de repositionner précisément le décor par rapport aux formes de relief et aux volumes sur et autour desquels semblent s'articuler les représentations - nous inscrivant ainsi, en somme, au cœur des problématiques actuelles de lecture et d'analyse de l'art pariétal, lesquelles soulignent le rôle manifeste du milieu physique dans l'exécution des tracés et le "paysage mental » des artistes paléolithiques. La finalité est, dans un avenir proche, de proposer un modèle numérique de terrain (Malaurent et al. 2005) et un fac-similé virtuel en trois dimensions, pour étudier le mode de décoration de la cavité et l'évolution de ses parois. Enfin, à la faveur de nouveaux types d'éclairages et de tirages photographiques de très haute définition, nous avons pu déchiffrer et expliciter quelques gravures inédites, généralement de lecture peu aisée en raison de l'état de surface altéré ou de la nécessité de choisir un angle d'éclairage particulièrement approprié.

4 La finalité d'ensemble qui a motivé la reprise de l'étude des gravures de la grotte du Sorcier était

1. de mieux cerner l'état sanitaire des parois (donnée qui peut évoluer rapidement),

2. de déterminer l'intensité des dégradations (naturelles et anthropiques) et leur impact,

3. de positionner précisément les unités graphiques dans l'espace de la grotte et les unes par rapport aux autres, et 
4. de fournir de nouveaux relevés - soit de représentations inédites, soit visant à préciser certains détails ou à proposer des lectures autres que celles jusqu'alors évoquées. Nous ne prétendons pas, à ce stade, à un inventaire exhaustif des panneaux gravés, mais présentons ici un bilan d'étape.

5 Trois aspects ont guidé notre analyse :

1. une tentative d'appréhension des gestes techniques mis en œuvre par les artistes préhistoriques ;

2. la relation de ces artistes au support rocheux; enfin,

3. l'impact des déprédations modernes affectant certaines gravures, qui participent de l'image offerte au préhistorien de ces représentations multimillénaires.

Dans l'état précédent des recherches (Delluc et al. 1987), on comptait vingt-huit représentations répertoriées, majoritairement figuratives, qui se répartissaient ainsi (fig. 1) : cinq chevaux, un bison, un bovidé, deux bouquetins, quatre animaux indéterminés, quatre anthropomorphes (le "Sorcier ", deux têtes humaines et une figure féminine schématique), neuf signes, deux traits isolés. G. Bosinski et J.-P. Duhard ont chacun cru récemment reconnaître de nouvelles représentations. Celles-ci sont en cours de publication par eux-mêmes. À l'issue des campagnes 2009, 2010 et 2011, nous avons découvert plusieurs gravures inédites (toutes ne font pas l'objet de cet article). Dans ce qui suit, les figures ne sont pas décrites dans l'ordre d'inventaire.

\section{Méthodologie : les techniques mises en œuvre en 2009-2010-2011}

\section{Sectorisation}

7 En 2009, nous avons divisé la cavité en plusieurs secteurs (figs. 2 et 5). Les parois ornées ont été subdivisées en panneaux, afin de clarifier la description ${ }^{4}$.

\section{Utilisation des moulages}

8 L'idée principale qui nous a guidés tout au long de la mission était de passer le minimum de temps dans la grotte et devant les parois. Aussi avons-nous commencé par travailler sur les quatre moulages conservés dans le petit musée attenant au site (fig. 3). Ceux-ci ont été réalisés par R. David dans les années 1970, à la demande du professeur H. de Lumley, sur les moulages de l'abbé Glory (David, communication orale du 16 novembre 2010). Il ne s'agit donc pas d'un des positifs réalisés sur le premier moulage de 1966, dont on pourrait craindre que la qualité et la précision aient diminué au fil des tirages, ni d'un moulage sauvage postérieur à 1966. Sa précision est donc remarquable, ce que nous avons pu vérifier face à l'original dans la grotte. Nous avons préféré travailler avec ceux-ci plutôt qu'avec les moulages Glory conservés à l'Institut de Paléontologie Humaine, car ils étaient sur place et facilement mobilisables. La comparaison du volume du moulage avec celui de la cavité a également permis de confirmer que la couche bleutée qui recouvre la paroi par endroits (fig. $\mathrm{A}^{5}$ ) correspond bien à la surface enduite par le produit démoulant en 1966. Détail curieux : une paroi non gravée est également recouverte de cette pellicule bleutée : s'agit-il de la trace d'un 
test réalisé avant l'opération principale, afin de s'assurer que le moulage était sans danger pour la paroi ? Ce qui permettrait d'en inférer que la couleur bleutée n'est apparue que plus tard; en effet, si elle était survenue dans l'instant, nul doute que les mouleurs auraient interrompu leur travail !

9 Travailler sur les moulages a donc réduit notre temps de présence dans la cavité et devant les parois les plus fragiles. Cela nous a permis également d'observer les tracés de manière plus confortable et de faire varier à loisir les angles d'éclairage.

\section{Méthode de relevé}

Figure 1 - Les représentations actuellement connues de la grotte du Sorcier (d'après Delluc et al. 1987).

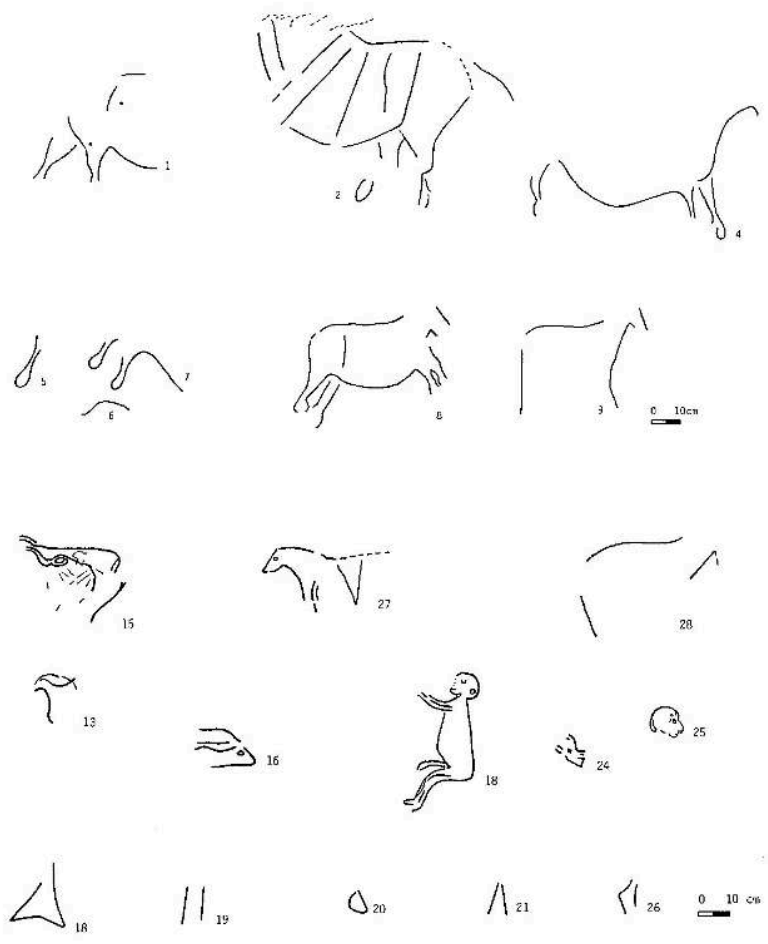


Figure 2 - Vue générale de la cavité et sectorisation (photo H. Paitier 2009).

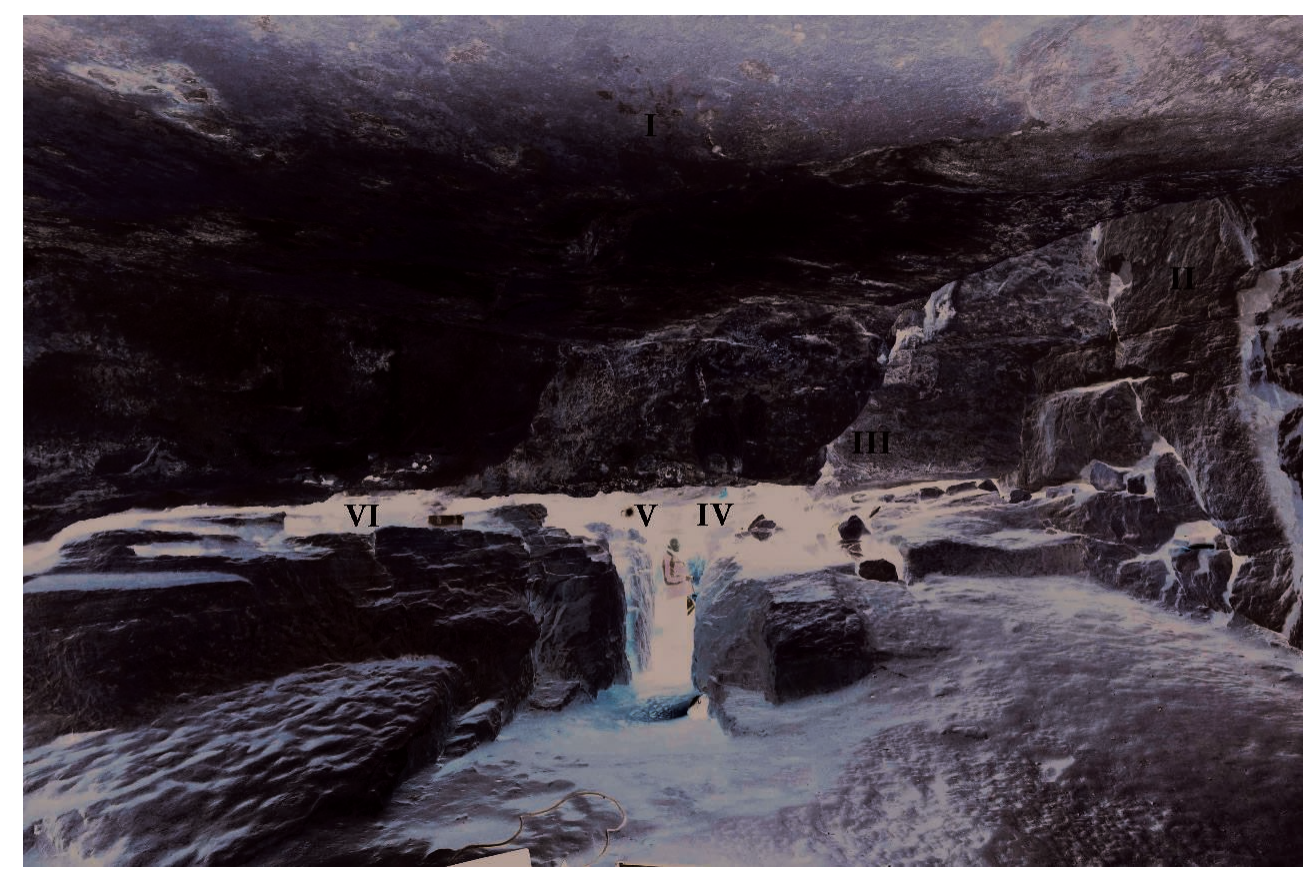

10 Nous avons privilégié la méthode de relevé sur photographie.

11 Les enregistrements photographiques se sont déroulés en trois séries de prises de vues. La première série s'est effectuée sur les moulages. Elle a consisté à faire tourner un éclairage autour de chacun des quatre moulages, afin de faire ressortir le maximum de gravures possible. Une photographie est prise tous les $30^{\circ}$ de rotation minimum, ce qui donne 12 éclairages différents par moulage (fig. 4) et met en valeur tous les tracés perpendiculaires à l'axe de la lumière artificielle.

12 Une seconde série de photographies a été faite dans la grotte avec l'aide des éclairages à fluorescence et a concerné des gravures ou panneaux distincts, le choix de l'emplacement des éclairages étant cette fois-ci beaucoup plus délicat vu la configuration de la grotte. C'est un assemblage de plusieurs images de la même figure qui permet d'obtenir un résultat très détaillé des gravures. Cette fois-ci encore, la position des éclairages variant, une multitude de tracés apparaissent, laissant croire à une superposition de plusieurs gravures difficilement interprétables chronologiquement.

13 La troisième série concernait le panneau du "Sorcier ». Pour cela, l'installation d'un rail de prise de vue en aluminium sur lequel coulisse l'appareil photo a été nécessaire. L'enregistrement a mis en évidence le problème de distance de mise au point. La distance du sujet doit être sensiblement la même pour réduire les déformations ; cela a particulièrement bien fonctionné sur le panneau du «Sorcier", mais a nécessité plusieurs balayages en fonction de la distance par rapport à la paroi de la grotte. Mais les différentes orientations des sources de lumière révèlent des gravures extrêmement complexes. Une difficulté est venue s'ajouter dans le fait que la paroi rocheuse absorbe énormément la lumière par son faible pouvoir réfléchissant, ce qui oblige à des poses plus longues que dans d'autres grottes. En effet, la roche est de couleur gris bleu foncé, noircie par la suie (la grotte ayant servi d'habitat à différentes époques: Delluc et al. 1987) et le temps, avec des éclats d'enlèvements apparaissant jaune orangé vif. Ces écarts de couleur et de réflexion obligent à doubler chaque photo (bracketing) afin de 
garder du détail sur toute la surface. Un système d'éclairage de plus petite taille doit être utilisé à l'avenir sur certaines figures pour certains recoins réduits.

\section{Levé topographique}

Les 23 et 24 novembre 2010, l'un d'entre nous (VP), assisté de M.-D. Pinel, du SRA Bretagne, a réalisé la couverture topographique de la cavité, et coté précisément les représentations. En accord avec le SRA, il a installé trois points topographiques fixes, dans le mur d'entrée et les aménagements modernes, ainsi que dans l'encaissant du secteur I, qui lui ont servi à trianguler précisément. Cette topographie minutieuse était nécessaire, surtout en vue de l'obtention d'un modèle numérique de terrain. Elle est par ailleurs venue corriger quelques points de la précédente topographie (figs. 5, B, C, D).

\section{Description des figures relevées pendant les campagnes 2010 et 2011}

\section{Bison $\mathrm{n}^{\circ} 1$}

\section{(Secteur II, paroi droite, panneau I : unité graphique 1)}

La première gravure profonde, à la limite du bas-relief pour certaines parties anatomiques, est un arrière-train de bison figuré en profil droit et en train d'avancer. La longueur est de $39 \mathrm{~cm}$ pour une hauteur de $23 \mathrm{~cm}$. Une description détaillée (Delluc et al. 1987 - p. 370) ainsi que deux relevés (ibid. ; Dams 1980) (fig. 6) ont déjà fait l'objet de publications.

Des enlèvements médiévaux - quatre trous précisément, dont on retrouve les pendants sur la paroi en vis-à-vis - ont fortement endommagé la paroi à proximité immédiate de la figure, que seul le trou inférieur gauche semble avoir épargnée. Le relief et le volume de la paroi sont particulièrement mis à profit: une fissure a servi de ligne de sol imaginaire ; la ligne de ventre est aussi en partie matérialisée par une fissure, et il en est de même pour la partie postérieure. La fesse, la patte arrière droite et l'aine sont bien individualisées; la patte arrière gauche est en position fléchie, travaillée dans sa partie antérieure. Un début de queue semble se dessiner, en partie entaillée par une fissure : soit l'artiste paléolithique a fait partir un bout de paroi, fragilisé par la fissure (le bison serait alors inachevé), soit cet enlèvement résulte des aménagements médiévaux. Patte arrière droite, ventre, partie supérieure de la patte arrière gauche, partie postérieure et croupe présentent un début de polissage.

Sur cette première figure, les reprises récentes, au fusain noir, ont surligné le creusement de la ligne de dos, une cupule naturelle qui a été détourée pour faire naître un œil, et une desquamation de la calcite de manière à créer un front et une corne (fig. 6). 
Figure 3 - Moulages des représentations 25 à 27 (en haut à gauche), du Bison 15 (au milieu à gauche), des représentations 23 et 24 (en bas à gauche) et du "Sorcier » 18 (à droite au milieu), réalisés par R. David à partir des moulages Glory de 1966 (photo H. Paitier).

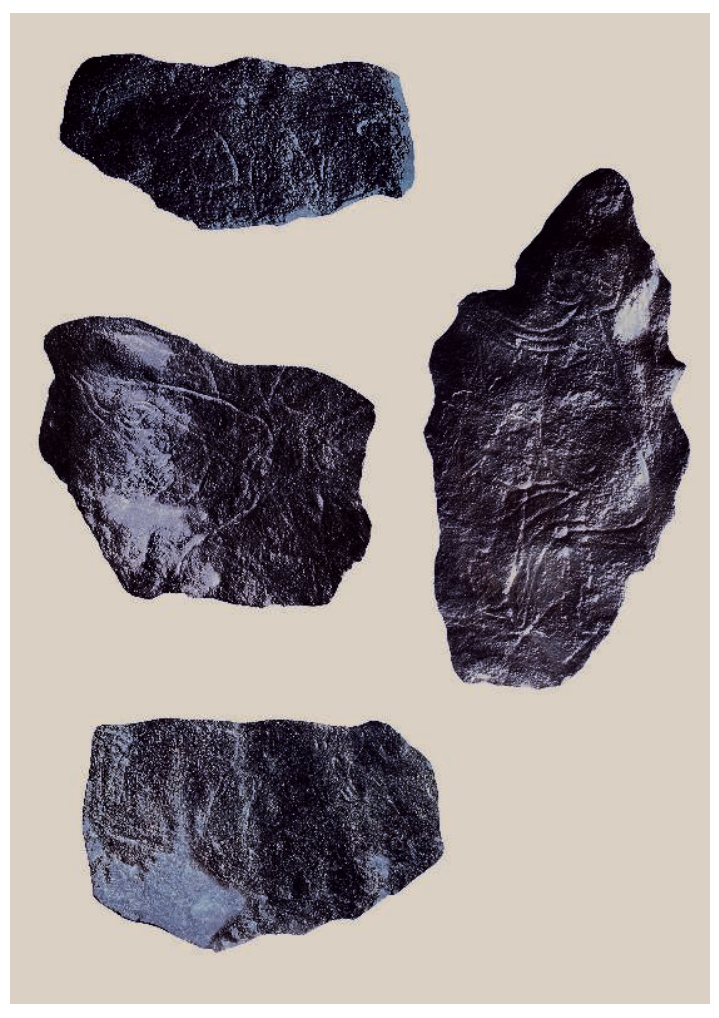

Figure 4 - Moulage du " Sorcier ». Planche montrant les différents tracés visibles suivant les directions d'éclairage indiquées par les flèches rouges (photos et montage H. Paitier).

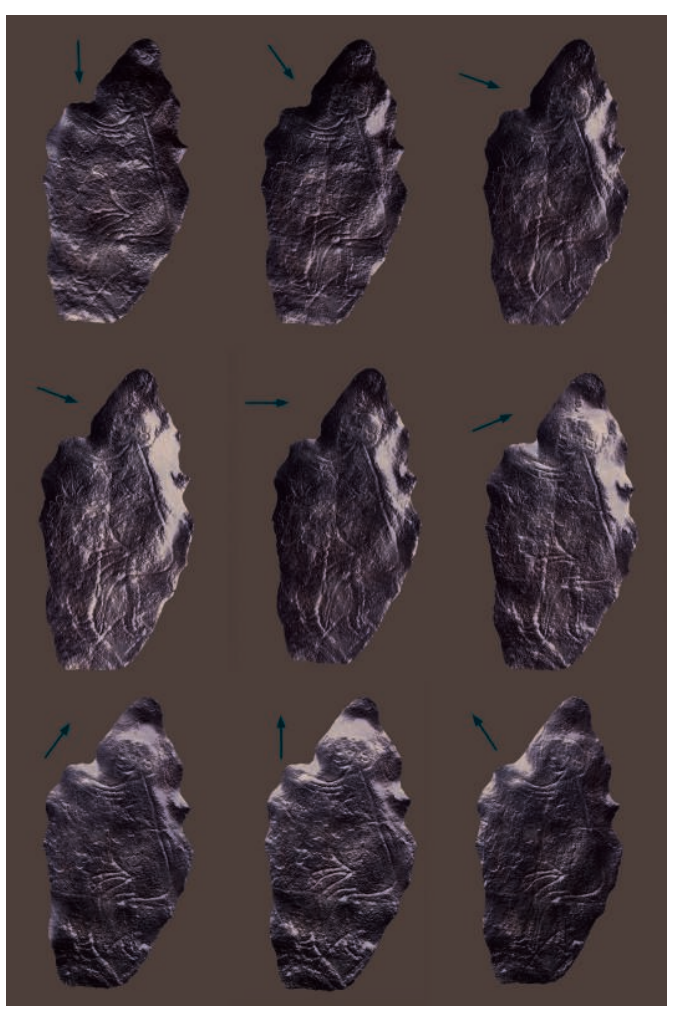


Figure 5 - Position et altitude des représentations de la grotte du Sorcier (levé V. Pommier et M.-D. Pinel).

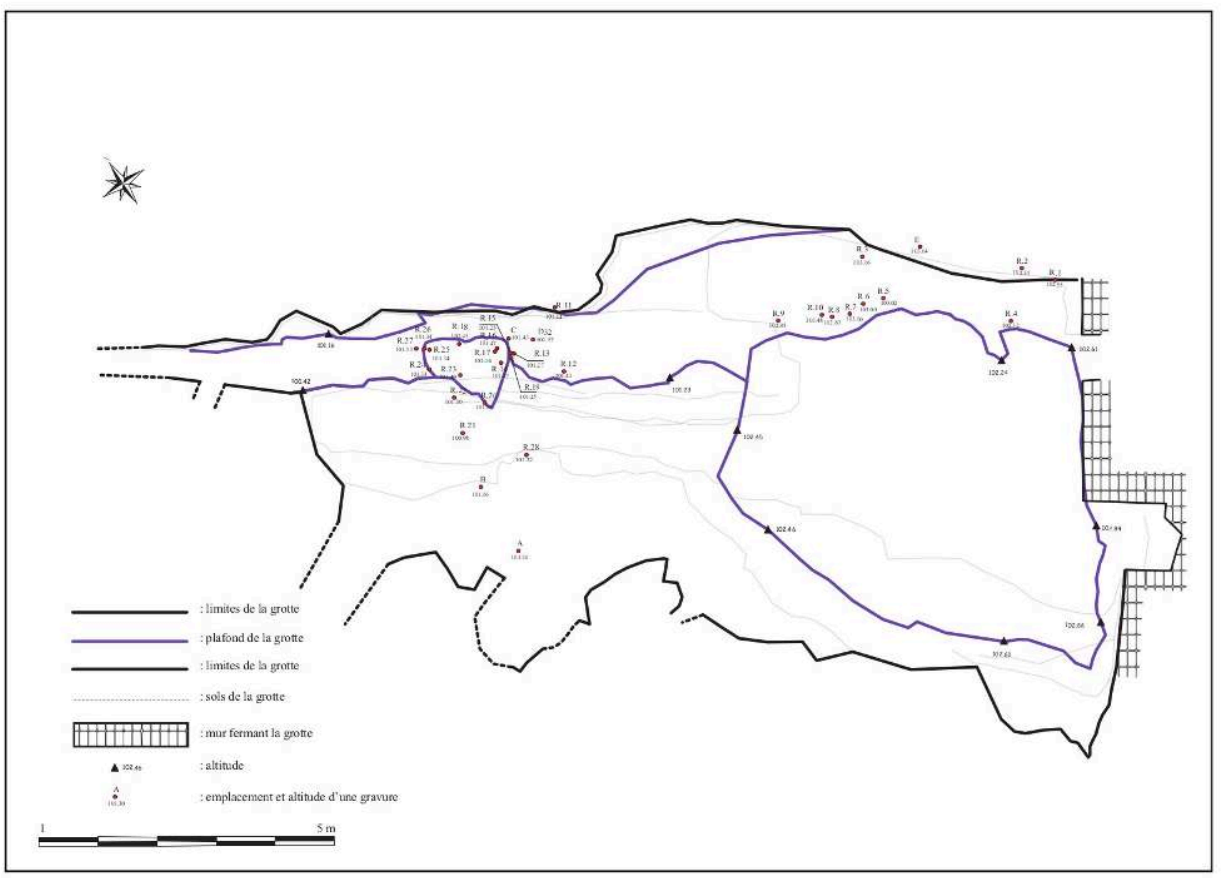

\section{Cheval $n^{\circ} 2$}

\section{(Secteur II, paroi droite, panneau I : unité graphique 2)}

Delluc et al. (1987 - p. 370) (fig. 7) ont déjà proposé une description détaillée de ce cheval apparemment sexué et orienté en profil gauche, en représentation conventionnelle de galop de type "canter " (ce terme désigne le galop d'essai d'un cheval de course, Reinach 1900), c'est-à-dire avec les postérieurs posés au sol et la partie proximale des membres antérieurs levée. Les sabots «en boule » rappellent la convention utilisée pour les chevaux de Lascaux (ibid.), quoique la représentation de l'épaule ne soit pas spécifique et se rencontre déjà sur des figurations d'époque gravettienne (tel le cheval $n^{\circ} 15$ de la grotte Mayenne-Sciences : Pigeaud 2004) (fig. 7).

La forme des reliefs au-dessus du garrot de l'animal a pu laisser penser qu'une tête retournée « en Agnus dei» avait été représentée - idée d'ailleurs qu'a eue l'auteur des déprédations modernes (les traits gravés de ce cheval ont été repassés au crayon graphite), puisqu'un enlèvement d'origine anthropique a été identifié à l'emplacement supposé de l'oreille. Mais nous n'étions pas convaincus par cette interprétation et étions décidés à résoudre cette question, par l'examen précis des tracés. Peu avant la fin de la mission 2011, il nous a ainsi semblé reconnaître les tracés d'une tête (non retournée) sous le voile de la calcite qui oblitère la paroi à main gauche; des observations plus poussées en lumière polarisée pourraient nous permettre d'approfondir l'étude. De la même manière, nous nous interrogeons à propos des cupules qui parsèment le corps du cheval: sont-elles d'époque paléolithique ou médiévale? Résultent-elles simplement de la corrosion? Une analyse des profils de creusement, après une numérisation 3D, semble tout indiquée pour apporter quelques éclaircissements. 
Figure 6 - Bison 1. a. Relevé analytique (d'après Delluc et al. 1987). b. Relevé Dams 1980. c. Photo $H$. Paitier. d. Relevé analytique. En rouge, les tracés modernes au fusain. En grisé, les enlèvements médiévaux. En marron, le mur actuel (relevé P. Bonic, DAO R. Pigeaud).

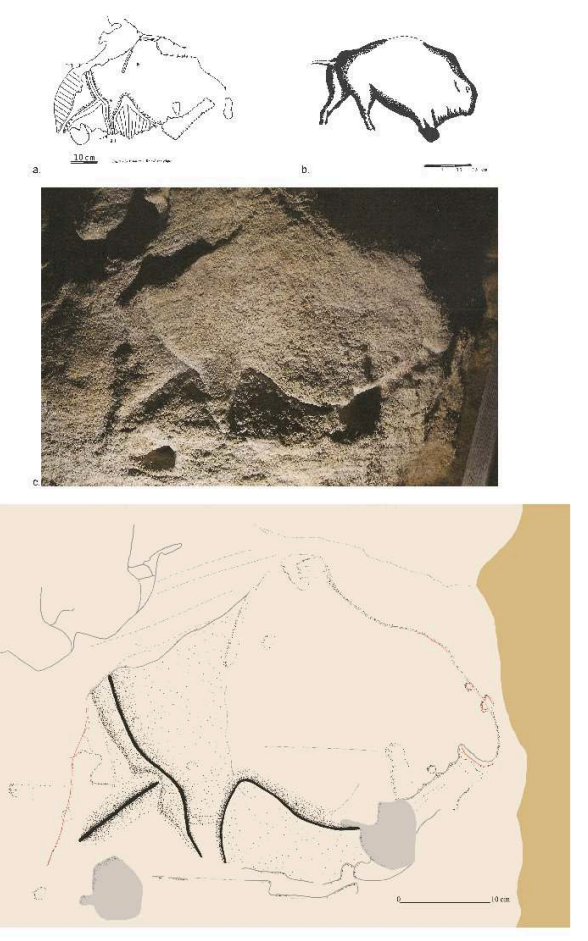


Figure 7 - Cheval 2. a. Relevé analytique (d'après Delluc et al. 1987). b. Relevé Dams 1980. c. Photo H. Paitier. d. Relevé analytique. En grisé, enlèvements de matières d'époque indéterminée. Lignes brunes : fil de la pierre. Lignes rouges : tracés modernes au fusain (relevé et DAO R. Pigeaud).

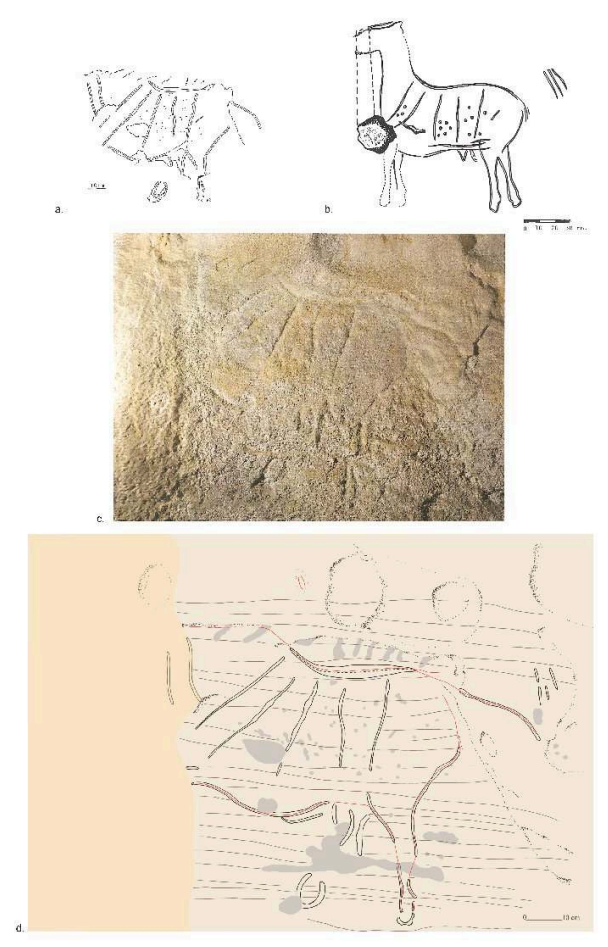

\section{Cheval $n^{\circ} 8$}

\section{(Secteur II, paroi gauche, panneau IV : unité graphique 8)}

Le cheval 8 a été étudié par B. et G. Delluc (Delluc et al. 1987, p. 374) (fig. 8). Il s'agit d'un équidé en profil droit, qui se trouve sur la paroi gauche de la diaclase formant le secteur II, à environ 2,70 $\mathrm{m}$ du sol actuel pour le bas de la figure, sur un surplomb rocheux à la surface presque verticale. Il est tourné vers la droite (i.e. vers le fond de la cavité) et mesure $50 \mathrm{~cm}$ de longueur pour $27 \mathrm{~cm}$ de hauteur. Il est situé entre deux trous profonds résultant vraisemblablement d'aménagements médiévaux (fig. 9).

Le contour du cheval est un bas-relief réalisé par la technique dite du "relief exhaussé » (Garcia 1989), connue notamment au Roc-de-Sers (Charente) et à Cap-Blanc (Dordogne) (Bourdier 2009-2010), qui a permis de dégager clairement les jambes, la ligne de ventre, l'arrière-train et une partie de la ligne de dos, avec une profondeur de trait variant de 8 à $20 \mathrm{~mm}$. Tout le bas de la figure a été obtenu par un piquetage qui précise les lignes de contour tout en modelant l'animal en relief. Comme l'ont fait remarquer B. et G. Delluc (1987), les impacts de piquetage sur cette partie sont encore bien visibles car le fond des parties abaissées n'a pas été lissé. On y voit des cupules résultantes, notamment au niveau de la ligne de ventre, bien arrondie et marquée par un tracé creusé par piquetage, large de 1,5 à $2 \mathrm{~cm}$; par endroits, apparaissent des impacts sous forme de courts traits, de 1 à $2 \mathrm{~mm}$ de largeur, qui semblent indiquer l'utilisation d'un outil plutôt fin et pointu, peut-être un burin. Les auteurs précisent également que le trait obtenu possède une section recticurviligne élargie, parfois à fond plat ou double fond. 
Les quatre jambes sont représentées en perspective bi-angulaire oblique (ou perspective semi-tordue), en extension formant une sorte de " galop volant ${ }^{7}$ » (Reinach 1900 - p. 218), dans une volonté d'indiquer le mouvement. Les jambes sont plutôt petites, courtes et minces, par rapport aux proportions d'ensemble de la figure. Pour les postérieurs, le bord externe est représenté en entier, se prolongeant par l'arrièretrain. Sa partie la plus fine est façonnée par des creusements assez abrupts, alors que plus on monte vers la ligne de ventre ou le haut de l'arrière-train, plus la profondeur du creusement s'atténue, modelant les contours. La face crâniale de la jambe est représentée bien en avant de la jambe arrière et n'est pas rattachée au corps de l'animal. Une réserve subsiste entre le haut de la jambe et la ligne de ventre pour évoquer la perspective - procédé que l'on retrouve notamment à Lascaux (Dordogne) et Chauvet (Ardèche). Elle est délimitée par un creusement abrupt sur tout son pourtour.

L'extrémité des jambes est arrondie. Les antérieurs sont représentés d'une manière moins réaliste que les postérieurs: ils sont presque en vue frontale, l'un à côté de l'autre, séparés par une surface creusée large d'environ $3 \mathrm{~cm}$. La jambe avant droite est plus courte que la gauche, ce qui peut être vu aussi comme une manière de créer une perspective et d'accentuer le mouvement, même si la nature du relief est telle qu'elle empêche de tracer un membre plus long: on se trouve en effet tout au bas du surplomb, là où la paroi s'efface. La maîtrise de l'artiste semble telle, cependant, qu'on peut supposer son intention d'exécuter la représentation à cet endroit, avec les possibles aberrations anatomiques qui en découlent. La jambe avant droite semble s'élargir vers le bas, peut-être pour suggérer le sabot.

Galop volant, membres petits et courts, réserve laissée en haut de la jambe arrière (Delluc et al. 1987, p. 374), ventre bien arrondi : autant de conventions graphiques qu'ont également adoptées les artistes qui ont peint et gravé les chevaux de la grotte de Lascaux (ibid., p. 374). Ces détails suggèrent pour nous l'existence de liens entre l'art des deux grottes, distantes de quelques dizaines de kilomètres seulement.

$\mathrm{Au}$ niveau de l'arrière-train, quelques traits longs, presque verticaux, finement gravés au fond de la partie creusée, sont visibles. Ils pourraient indiquer une queue, même si visuellement l'œil a du mal à ne pas en voir une dans les parties brunâtres laissées en relief juste à l'arrière de l'animal. La zone à la gauche de cette surface est cependant abîmée, avec quelques traits fins d'aspect frais et non patinés qui semblent donc modernes, ce qui empêche toute lecture définitive de cet élément anatomique. En remontant vers le haut de l'arrière-train, on remarque un blanc entre le haut de la surface creusée délimitant la cuisse et le début de la ligne de dos : la ligne n'a pas été marquée et s'interrompt (fig. 9). Celle du haut de l'arrière-train reprend ensuite, marquée par une dépression assez profonde $(1$ à $1,5 \mathrm{~cm})$ surmontée par un léger surplomb de la paroi. Elle s'interrompt de nouveau avant de reprendre jusqu'au niveau de l'encolure. Cette partie du tracé a un aspect très différent de celui du ventre: la surface semble lissée, "brossée ", et ne comporte presque pas d'aspérités ou de ces reliefs brunâtres qui sont les restes de la paroi d'origine.

La prudence est de mise avec une partie du trait qui a été repassée au doigt, au crayon graphite et avec un objet pointu à l'époque moderne, ce qui entrave considérablement la lecture de cette zone. Il apparaît cependant que ces reprises n'ont pas entamé profondément le tracé ni créé de relief profond ; l'auteur semble avoir voulu souligner ce qui n'était pas assez visible à son goût, peut-être dans l'intention de rendre la figure plus lisible vue du sol. Aussi la tête a-t-elle été entièrement rajoutée : on discerne à 
présent une ligne de crinière et de dos, une oreille, une reprise du chanfrein, le tour du bout du nez clairement marqué ainsi que le départ de la ligne de poitrail (fig. E). Ces traits sont tantôt gravés finement, tantôt plus largement, comme faits au doigt ou au bâton ${ }^{8}$, et tous sont nettement modernes et d'une couleur claire. Comme l'avaient relevé B. et G. Delluc (ibid., p. 374), seule une dépression profonde avait été façonnée par les Préhistoriques à l'emplacement probable du chanfrein, sur la partie gauche de laquelle passe le tracé du «nouveau » chanfrein. Associée à l'effet du relief sur la paroi, cette dépression suffisait à suggérer une tête au cheval sans que la crinière soit individualisée, même si d'autres traces préhistoriques n'ont pas été reconnues dans cette zone.

D'autres tracés encore visibles devant le poitrail de l'animal permettent cependant d'envisager une autre possibilité, celle d'un cheval à tête baissée vers l'avant. On voit en effet à l'avant une ligne subverticale profonde, presque droite, jusqu'alors associée au cheval 9 comme partie de sa jambe arrière. Vers le bas de cette ligne, quelques centimètres avant l'arête du surplomb rocheux, on remarque, partant de son bord vers la gauche, un abaissement de la paroi qui permet de dégager et de modeler l'arrondi d'un museau - tracé qui se prolonge ensuite pour former le menton et le cou. Ce modelé des bords apparaît nettement sous un éclairage judicieusement orienté, ainsi que l'abaissement de la surface entre la jambe avant du cheval et la tête, un fond de trait soulignant le bord des tracés. Notons que la ligne subverticale profonde ne semble pas devoir être associée à cette tête car les proportions ainsi obtenues seraient aberrantes. Un tracé plus léger, oblique, très ténu, pourrait plutôt correspondre à ce chanfrein même si, comme l'ont fait remarquer B. et G. Delluc (ibid., p. 374), la plus grande prudence est de mise avec une telle surface, couverte de cupules coalescentes pouvant évoquer des traits anthropiques (fig. E) : l'avant-main du cheval se trouve dans une zone altérée, ce qui empêche de faire la jonction et de confirmer cette hypothèse, même si ses proportions la rendraient plausible. Une autre possibilité est celle d'un reste de figure antérieure au cheval 8 , ou encore d'une reprise de la figure à un moment donné.

D'autres traces existent (voir infra) qui indiquent une première étape de travail de la paroi avant la réalisation du cheval 8. Cette dernière représentation a été qualifiée de " pommelée " par B. et G. Delluc (ibid., p. 374) en raison de l'aspect de la surface du corps, qui présente une alternance de taches sombres et claires semblant pour la plupart résulter essentiellement des diverses altérations pariétales; quatre d'entre elles, des cupules parfaitement circulaires et assez profondes (jusqu'à $1 \mathrm{~cm}$ ), paraissent anthropiques. La robe de l'animal est surchargée de cinq traits verticaux à subverticaux assez larges (de 0,6 à 1,2 cm), d'inégales longueurs, celui de gauche étant le plus marqué, ainsi que d'un trait plus fin au niveau de l'épaule. Ils sont répartis sur l'intérieur du corps sans qu'aucun ne touche les lignes de contour. Ces tracés rappellent ceux visibles sur le cheval « rayé " $\mathrm{n}^{\circ} 2$, également profondément gravé dans le même secteur de la grotte sur la paroi opposée. On remarque également une ligne gravée large de 3 à $4 \mathrm{~mm}$, grossièrement parallèle à la ligne de ventre, à l'intérieur de l'animal, depuis la jonction de la jambe arrière et de cette ligne de ventre jusqu'au niveau de la première jambe avant. Enfin, une ligne profondément gravée est bien visible sur l'arrière-train, avec son bord droit plus abaissé que le gauche. Elle ne semble correspondre à aucun détail anatomique ni à aucune convention habituelle, ce qui nous a poussés à inspecter attentivement la paroi aux abords immédiats de ce tracé. Nous y avons trouvé d'autres traits beaucoup plus ténus, qui semblent former au final la tête 
d'un autre animal, qui ne nous paraît pas être un cheval en raison de son arrondi et de sa compacité (fig. 9); peut-être s'agit-il des vestiges d'une figure antérieure à la représentation du cheval 8 et oblitérée en quasi-totalité par ce dernier, ce qui nous conduit à admettre l'existence de deux phases successives d'ornementation de la paroi dans ce secteur-là. Enfin, d'autres gravures fines visibles à l'arrière-train et au niveau de la tête de cheval soulignée récemment paraissent modernes.

Figure 8 - Cheval 8 et animal 9. Trait $\mathrm{n}^{\circ}$ 10. a. Relevé analytique (d'après Delluc et al. 1987). b. Relevé Dams 1980.
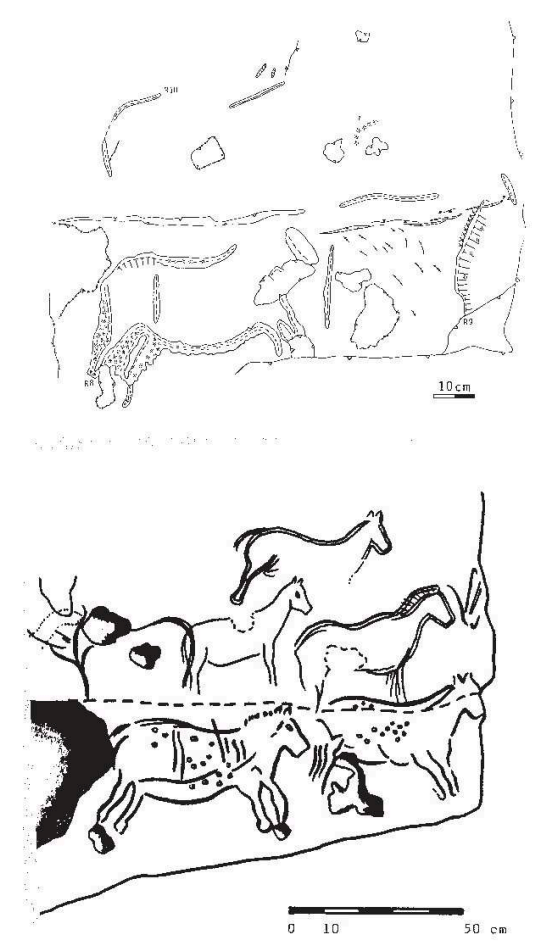
Figure 9 - a. Photographie du cheval 8 (à gauche) et de l'animal 9 (à droite) (H. Paitier). b. Cheval 8. Relevé analytique. Les traits rouges signalent les transformations modernes (frottements souvent arrondis, comme s'il s'agissait d'une brosse utilisée pour nettoyer la paroi ou effacer des traces, ou gravure ou frottements linéaires, ou pour tracer une nouvelle tête avec son toupet et son encolure (relevé E. Bougard). c. Animal indéterminé 9. Relevé analytique. En grisé : enlèvements de matières d'époque indéterminée. Lignes brunes : fil de la pierre. Traits rouges : tracés modernes. Jaune : arrachements récents (relevé E. Bougard, DAO R. Pigeaud).
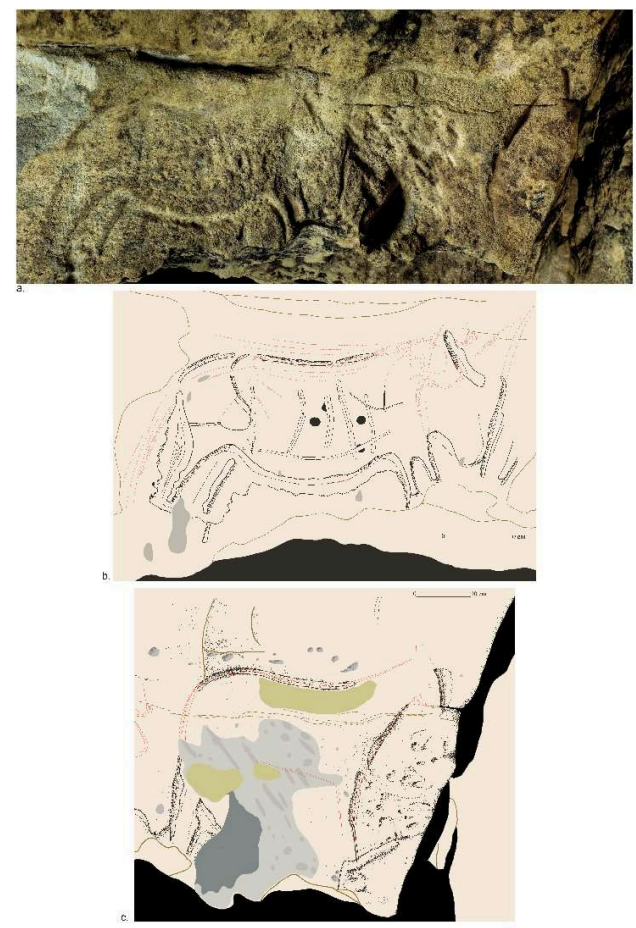

\section{Animal indéterminé $\mathrm{n}^{\circ} \mathrm{9}$ (Secteur II, paroi gauche, panneau IV : unité graphique 9)}

Cette figure est appelée « cheval $n^{\circ} 9$ » dans les études précédentes (ibid., p. 374) et est exécutée à environ $2,70 \mathrm{~m}$ du sol actuel, sur un surplomb rocheux à la surface presque verticale, à droite du cheval $n^{\circ} 8$ (voir supra). Elle est aussi en profil droit, et s'inscrit dans un rectangle de $45 \mathrm{~cm}$ de long et $41 \mathrm{~cm}$ de haut. La technique mise en œuvre est la sculpture en bas-relief et la gravure (profonde à fine). L'étude précédente (ibid. p. 374) avait abouti au relevé de quatre larges traits formant le contour d'un animal interprété comme un cheval, que voyait déjà L. Dams (1980) (fig. 8 et 9). Ces quatre tracés principaux forment un contour sommaire et incomplet: une ligne droite de $16 \mathrm{~cm}$ à gauche, verticale, pour la queue ou le bas de la patte arrière ; après un blanc, un trait bien marqué, de $29 \mathrm{~cm}$ de long à la corde, qui part du haut de la cuisse et forme la croupe et le dos jusqu'à la crinière; un simple trait subvertical de $8 \mathrm{~cm}$ pour la tête, large de 1 à $2,5 \mathrm{~cm}$ et profond de $1,5 \mathrm{~cm}$ au niveau du chanfrein; enfin, le poitrail et le bord de la patte avant sont figurés par ce qui semble, au premier abord, une seule ligne longue de 32,5 cm, subverticale et légèrement incurvée, partant du haut de l'encolure.

Le nouveau relevé effectué en 2011 est venu compléter les observations précédentes en précisant certains détails (fig. 9). Ainsi, le trait droit vertical mentionné précédemment a une largeur totale irrégulière $(0,5 \mathrm{à} 2 \mathrm{~cm})$ mais le fond de trait est rectiligne et fin, 
signe d'un outil pointu. Il s'interrompt au bord du support rocheux. La surface immédiatement à sa droite porte des traces de travail sur une dizaine de centimètres, non signalées dans les études précédentes : un trait subvertical de $5 \mathrm{~cm}$ de long et $1 \mathrm{~cm}$ de large, dont l'extrémité atteint le bord du surplomb vers le bas à $3 \mathrm{~cm}$ de l'autre trait et dont la partie haute rejoint une zone modelée en creux ( 2 à $3 \mathrm{~cm}$ de haut, $7 \mathrm{~cm}$ de large, profondeur maximale de $1,2 \mathrm{~cm}$ ) interrompue par le trou médiéval. Ces éléments peuvent être interprétés comme figurant une patte fine qui s'élargit en formant le départ de la ligne de ventre et de la cuisse. Il n'y a pas de jonction visible avec le haut $\mathrm{du}$ trait de gauche, mais cette ligne correspondrait alors à une queue longue et fine. En partie basse à droite, nous avons repéré de nouveaux tracés, bien visibles avec un éclairage venant de la gauche. Le bord du relief ainsi formé est indiqué par un trait creusé subvertical de $4 \mathrm{~cm}$ de long qui part du bord du surplomb, poursuivi à droite par une sorte de relief long de $12 \mathrm{~cm}$ et large d'entre 1,5 et $2 \mathrm{~cm}$, subhorizontal (fig. 10).

Ces nouveaux éléments, relevés en partie inférieure de la figure, s'agencent nettement pour évoquer, selon nous, le bas du corps d'un bison à petites pattes et grand corps massif, au fanon bien indiqué par un tracé presque horizontal - de type Font-deGaume, par exemple. Dans cette optique, nous avons réexaminé l'ensemble des traits. Sur la partie avant, à l'emplacement supposé de la tête, nous n'avons pas pu déceler de traces probantes de façonnage, même si le volume d'ensemble des surfaces est tout à fait évocateur de cet animal. La ligne de dos actuelle pourrait correspondre à celle du bison, à moins que celle-ci n'ait été plus basse, dans la zone à présent détruite ; le trait décrit comme le chanfrein du «cheval $n^{\circ} 9$ ", à l'avant de la bosse du dos. En outre, le bord du surplomb rocheux ayant été utilisé, on ne peut exclure une intégration des reliefs naturels.

En revanche, la ligne formant l'avant du poitrail du « cheval » pose problème avec cette interprétation : elle entaille profondément la figure et ne parait pas pouvoir constituer la corne ni aucun autre détail anatomique. Ce trait est en fait composé de trois parties distinctes. Vers le haut, on peut véritablement parler de sculpture, avec un abaissement du bord extérieur de presque $2 \mathrm{~cm}$ par endroits, donnant un tracé bien rectiligne et abrupt qui s'élargit et s'adoucit sensiblement dans sa partie basse et met en valeur le modelé du corps; ce trait s'interrompt ensuite brièvement, même si l'œil fait se poursuivre la ligne vers le bas. La suite consiste en un tracé plus large ( $1 \mathrm{~cm}$ environ), aux bords moins abrupts et en creux ; une ligne gravée plus fine le prolonge vers le bas, qui entaille le relief (possible fanon du bison?).

À l'image d'autres figures, celle-ci a été dans un premier temps endommagée par les troglodytes médiévaux, qui ont façonné un large trou de poutre en son milieu et abîmé ainsi à coups de pioche toute la zone centrale de l'animal. Elle fut ensuite repassée ou complétée après l'étude menée par B. et G. Delluc (op.cit. Delluc et al.), au crayon à papier, avec un objet pointu, au doigt ou avec un bâton au bout arrondi. Ainsi, le fond du trait n'est plus lisible au niveau des deux parties basses du « bison ».

Nous proposons de voir sur cette paroi deux phases successives d'ornementation, avec une figure initiale de bison postérieurement retravaillée en un autre herbivore (cervidé ou bouquetin). Nous ne pensons pas que ce dernier soit un cheval, notamment par comparaison avec le cheval voisin $n^{\circ} 8$ (voir supra), d'un style très différent (idée de mouvement, détails anatomiques, travail moindre de rendu du volume) quoiqu'il existe des similarités, à l'image de cette tête simplement suggérée par une ligne de chanfrein. Il ne nous est malheureusement pas possible de prouver la contemporanéité de la 
réalisation des figures - condition essentielle des remarques qui précèdent; remarquons néanmoins que les deux représentations semblent former un panneau et se suivent sans se chevaucher, signe possible que l'on aurait affaire à une composition. Rappelons enfin que nous avions aussi remarqué des traces correspondant à une phase de travail plus ancienne sur le cheval $n^{\circ} 8$ (voir supra). Une reprise de toute cette partie de la paroi n'est donc pas à exclure.

Figure 10 - Animal indéterminé 9 : photo de détail de la tête surlignée en blanc (photo H. Paitier, DAO A. Redou).

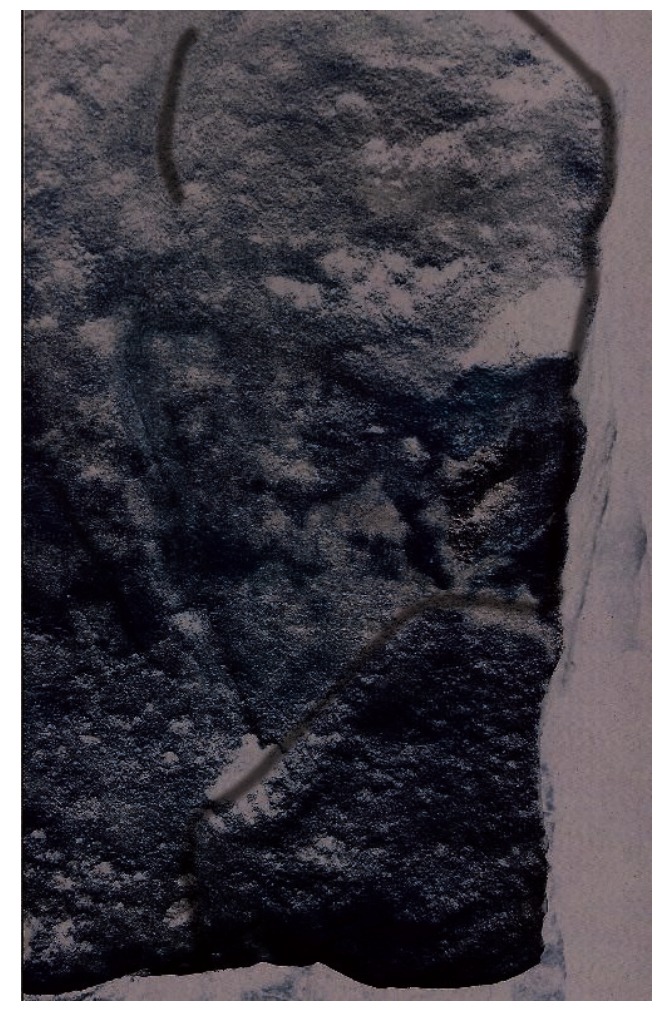

\section{Groupe de représentations $\mathrm{n}^{\circ} 25$ à 27 (Secteur III, paroi gauche, panneau VIII : unités graphiques 25 à 27)}

Située sur une lame rocheuse subverticale en paroi gauche de la diaclase s'étirant vers les profondeurs de la grotte à main droite, peu après le panneau du «Sorcier ", cette composition s'inscrit dans un rectangle de $50 \mathrm{~cm}$ de long sur $15 \mathrm{~cm}$ de haut. Le support calcaire est altéré, partiellement recouvert de placages argileux par endroits et marqué par des dépôts de gypse encadrant à l'horizontale les figurations gravées, soit par des ponctuations franches bien circonscrites sur la paroi, soit par des plages plus diffuses (bandeau supérieur); côté droit, le bas de la composition voit l'avancée d'un recouvrement de gypse plus soutenu; le départ d'une écaille mesurant $8 \mathrm{~cm}$ dans sa plus grande diagonale pour $5 \mathrm{~mm}$ d'épaisseur y a également emporté la roche en surface.

La représentation 25, longue de $10 \mathrm{~cm}$ et haute de $10 \mathrm{~cm}$, décrite par B. et G. Delluc comme une "tête humaine au visage bestialisé » (fig. 11), recoupe en partie une concavité de la roche (ibid., p. 385). 

trait d'une extrême finesse »; malheureusement, les traits ont été récemment repassés au moyen d'un outil pointu, les rendant par là même beaucoup plus visibles - l'outil ayant entamé la surface partiellement recouverte d'efflorescences de gypse a fait réapparaître le placage argileux. Ces reprises n'ont pas été d'une grande précision, ni toujours d'une grande habileté : en effet, le contour du profil est restitué au moyen de segments de traits subrectilignes, se recoupant légèrement aux extrémités, et s'écartant parfois du tracé d'origine. Du sommet du front à la glabelle (où l'extrémité du nouveau tracé présente des particules arrachées à la roche), on a ainsi ignoré la courbure pour relier ces deux points par un tracé rectiligne. De même, l'œil, initialement arrondi, apparaît à présent en forme d'amande car le bord supérieur gauche n'a pas été repris. Par ailleurs, en raison du prolongement du geste moderne ou d'une méconnaissance partielle des tracés paléolithiques, des traits nouveaux, n'excédant pas $1 \mathrm{~cm}$ de long, ont pu être relevés : à la base de l'arrière-crâne, près de l'appendice nasal (au possible emplacement d'une bouche) ou encore à l'arrière de l'œil. Les tracés uniquement paléolithiques demeurent résiduels. selon nous, une «silhouette féminine, ou plutôt gynoïde, tournée vers la droite " (FFS suivant leur terminologie: Delluc et Delluc 1995). Cette figure, longue de $2,8 \mathrm{~cm}$ et haute de $5,5 \mathrm{~cm}$, d'un graphisme géométrique, a aussi été repassée assez profondément. L'intervenant moderne a fait se croiser ses reprises sur quelques millimètres au niveau de la jonction entre la ligne de dos et la courbure de la fesse.

Gelluc avaient décrit pour la représentation 27 , longue de $35 \mathrm{~cm}$ et haute de 15 $\mathrm{cm}$, une «avant-main de cheval probable [...] tourné[e] vers la gauche» (Delluc et al. 1987 - p. 388). À la nouvelle lecture de celle-ci, il nous apparaît que ce cheval est plus complet, puisque les lignes de dos et de ventre ainsi que le départ d'une jambe arrière sont gravés. La ligne de dos, qui épouse les formes du relief, est plus marquée au-dessus du négatif d'écaille - par deux tracés parallèles. Au niveau de l'ensellure de l'animal, la lecture est rendue difficile par un enchevêtrement de tracés larges ou plus fins, organisés en faisceaux sécants, au point qu'il est délicat de décrire précisément la position de cette ligne de dos. La ligne de ventre était également déjà fortement suggérée par le relief naturel; à l'extrémité gauche de la dépression, des tracés fins anthropiques la prolongent, validant selon nous l'intégration de ce relief par l'artiste préhistorique. Ce tracé est aujourd'hui atténué en raison du dépôt de gypse.

Là encore, la nuque, une partie de la ligne de dos, la tête, l'œil, le poitrail et le départ des antérieurs, ainsi qu'une possible oreille, ont été repris par un tracé fin et profond. Des particules d'argile signant cette reprise de traits sont encore visibles au niveau de cette oreille. Pour la bouche, peut-être pour bien marquer la courbure, on semble s'y être repris à deux fois, puisque deux incisions parallèles modernes sont visibles. En outre, la personne a semble-t-il été trompée, sur le poitrail, par « des traits en ogive et en trident évoqu[a]nt [...] un rendu malhabile de l'épaule et du membre antérieur ", selon l'interprétation de B. et G. Delluc (ibid.), dont il a aussi repassé les bords, en sorte que l'œil de l'observateur n'accroche pas immédiatement l'allure générale du cheval. Quant aux «traits en ogive ", dont le tracé central, vertical, recoupe très légèrement la branche de gauche et pourrait suggérer un sillon vulvaire, ils ne sont pas sans évoquer une représentation pubienne - un trait fin légèrement concave relie les branches latérales à $8 \mathrm{~cm}$ de leur jonction.

PALEO, 23 | 2012 
41 Le support très altéré, les dépôts argileux et gypseux (et non une altération de surface), le caractère alvéolaire du calcaire ne facilitent pas la lecture des terminaisons de traits. Plusieurs segments entrant dans la composition du panneau semblent ainsi se prolonger au-delà des traits repris à l'époque moderne : c'est notamment le cas des jambes avant du cheval (réalité anatomique : tracé des membres et/ou du muscle ?), de son chanfrein (existence d'une autre gravure sous-jacente, trop altérée ou que nous n'avons su déchiffrer encore ?), de la possible vulve gravée ou encore des deux tracés formant la figure gynoïde (rectiligne vers le haut pour le tracé de gauche, recourbé vers le bas pour celui de droite, voir Bosinski 2011). Ces tracés que nous considérons comme anthropiques sont très atténués, leur lecture rendue possible par un éclairage de type lumière du jour particulièrement rasant.

42 Enfin, le même éclairage révèle de nombreuses incisions fines organisées en faisceau sur la gauche du panneau - l'un orienté sud-ouest/nord-est, recoupant le profil humain bestialisé dans sa partie inférieure, l'autre de moindre densité et subhorizontal, s'étendant de la base du pendant rocheux à l'aplomb du profil humain jusqu'à la jonction de la ligne de ventre du cheval avec le bord supérieur de la plage de gypse. Dans la partie centrale, ces tracés fins semblent inorganisés, sans orientation préférentielle. Il ne nous a pas été possible de proposer de lecture de ces tracés fins. Par ailleurs, quelques traits à partir de la tête bestialisée semblent prolonger la gravure sur le pan arrière de la lame rocheuse - secteur qu'il nous faudra attentivement déchiffrer. Signalons que les tracés se prolongent sur l'autre face de la lame rocheuse et qu'il conviendra d'élaborer un système (miroirs ?) pour parvenir à déchiffrer ce côté, difficilement atteignable pour le moment en raison de l'étroitesse du passage et la fragilité de la paroi (fig. 11c).

\section{Panneau du "Sorcier » : nouvelle lecture de la représentation $n^{\circ} 18$ (Secteur IV, voûte, panneau IX : unité graphique 18)}

Ce panneau extrêmement complexe se déploie sur une grande voûte. Nous avons privilégié pour le moment le relevé de la languette rocheuse qui comporte les représentations 14 à 18, suivant l'inventaire de B. et G. Delluc (Delluc et al. 1987). Nous nous sommes attachés au déchiffrement des nombreux tracés, aidés en cela par le moulage (fig. 3) car, malheureusement, la progression du gypse, qui non seulement recouvre mais aussi altère la paroi, ainsi que les actes de vandalisme ont rendu certains tracés difficilement lisibles. En ce qui concerne les enlèvements de matière, qui laissent une trace jaune, la comparaison avec le moulage, qui conserve le négatif de ces enlèvements et anciens clichés, démontre que ceux-ci sont anciens et n'ont pas évolué outre mesure. De ce côté-là au moins, la paroi n'a pas trop changé. Nous ne traiterons ici que de la représentation $n^{\circ} 18$, et nous publierons prochainement l'étude complète du panneau après relevé détaillé.

L'anthropomorphe surnommé familièrement le "Sorcier" a fait couler beaucoup d'encre (fig. 12). D'abord perçu comme un félin par les premiers découvreurs (Blanc 1955), il a ensuite été interprété comme un ithyphallique, interprétation renforcée par la figure similaire de Sous-Grand-Lac (Dordogne). Pour l'abbé Glory, le « Sorcier » était même un chamane, tenant dans ses mains un "sac aux esprits » ou " ongones » (Glory 1964). Jean-Pierre Duhard refuse l'interprétation d'anthropomorphe, en particulier à cause de la position anatomiquement aberrante du sexe (Duhard 1995). Jean Airvaux, 
arguant de la grosse tête et de la position fœtale du personnage, a proposé récemment de le lire comme la représentation d'un nouveau-né, le phallus étant un cordon ombilical (Airvaux, communication orale). En ce qui nous concerne, la comparaison des trois relevés existants et de la paroi n'a pas cessé de nous interpeller, ne serait-ce que par la multitude de traits inventoriés, qui semblent apparaitre puis disparaitre de façon anarchique. Technologiquement, cela nous semblait aberrant, l'usure de la paroi n'expliquant pas tout. Nous avions l'impression que les tracés avaient été relevés sans prendre en compte la gestuelle. Nous avons donc souhaité refaire un relevé précis, en poursuivant les départs de traits et en recherchant la logique et la chronologie des gestes.

Par ailleurs, deux détails nous ont troublés : la position du sexe et la position penchée du corps. Nous souvenant que cette figure avait d'abord été prise pour un félin, nous nous sommes demandé si nous n'étions pas en présence d'un quadrupède transformé en anthropomorphe. Le sexe serait un ancien autopode. En fait, l'œil, habitué à la lecture traditionnelle, ne chercherait pas à aller plus loin, orienté en quelque sorte par la configuration actuelle des lieux. Car il ne faut jamais oublier que nous sommes en présence d'une voûte: le sens de lecture peut avoir été multiple au cours du temps (figs. 13, F, G).

Deux autres éléments nous ont perturbés :

1. 1. La lecture actuelle du visage, en fonction de l'écaille de la paroi qui aurait servi à terminer le tracé du profil, ce qui nous a toujours semblé trop beau pour être vrai. Une longue pratique des grottes ornées et du relevé nous a enseigné que, si les utilisations du relief existent, il ne faut les envisager qu'en dernier recours : l'argument qui consiste à dire que le tracé est terminé par la fissure ou le bord rocheux ou l'écaille ne doit être privilégié que si aucun tracé n'est perceptible. Or, ce n'est pas le cas ici (fig. 13).

2. Nous sommes en présence d'un volume très perturbé par une succession de niches et de retours. Jusqu'ici, le panneau du "Sorcier " semblait avoir été conçu comme un espace bien délimité. Or, une observation attentive de la paroi montre que les tracés se poursuivent au-delà de la languette rocheuse. Le retour rocheux est très étroit. Pour en faire le relevé, il conviendra de trouver un système de miroirs qui permette de lire et d'effectuer des prises de vues qu'il faudra redresser ensuite (fig. $\mathrm{H}$ ).

47 Parlons d'abord de l'état de la paroi. Ainsi qu'il est possible de le voir sur le relevé analytique, les dépôts de gypse sont surtout concentrés sur les bords et les arêtes. D'après les anciens clichés, ils semblent n'avoir pas trop progressé; en revanche, une observation rapprochée montre qu'ils entament la couche extérieure de la pierre et en font une sorte de « mélasse » extrêmement fragile (fig. 14).

Autre élément : les arrachements de paroi, en jaune (fig. 14). Là encore, la comparaison avec le moulage (où leur profil figure) montre qu'ils n'ont pas progressé depuis 1966, sauf pour quelques petites lentilles. Ils se concentrent sur le relief principal de cette languette rocheuse, qui a un profil en forme de carène de bateau inversée. Ce qui signifie, selon nous, que cette partie de la paroi, la plus basse donc, était la plus proche du remplissage et a dû subir des frottements, et a contrario, que la partie la plus haute n'a pas dû être en contact avec le remplissage et est probablement restée dégagée, contrairement à la cuvette qui se trouve à la base de la voûte.

Leur lien avec les traits gravés est par ailleurs fort intéressant. Par exemple, il est à noter que le tracé du ventre est coincé entre deux enlèvements de matière et que le fantôme du tracé subsiste souvent dans l'écaille. Ce qui signifie non seulement que le 
trait gravé était profond, mais aussi que l'enlèvement de matière ne s'est pas fait brutalement, mais au fil du temps ou suite à un écaillage superficiel. Deux exceptions cependant : les enlèvements profonds et sans tracé qui se situent à la base de la verge et au niveau du genou gauche. Ici, en revanche, il semble bien que la paroi ait été altérée par un objet contondant, ainsi que cela s'est produit pour l'œil de la «lionne » bien connue des Combarelles I.

Pour ce qui est des déprédations modernes (figs. 14 et I), elles sont de deux sortes : les projections d'argile, dues selon nous à des coups de coude ou d'épaule de visiteurs indélicats, et les repassages de traits (crayonnages), nettement visibles parce que plus sombres, que l'on peut voir sur l'anthropomorphe, le bouquetin 16 et le bison 15. Pour ce dernier, on s'est contenté de repasser la narine, le museau et l'arcade sourcilière. Pour le bouquetin, on a redessiné approximativement le contour de l'œil, en le faisant davantage arrondi. Mais c'est l'anthropomorphe qui a le plus souffert: le vandale a accentué le contour de l'œil droit, « l'oreille » dans l'interprétation traditionnelle, ainsi que celui du front et de l'arcade sourcilière de l'œil gauche, créant ainsi une nouvelle tête qui n'a plus rien à voir avec l'ancienne. Il a également creusé l'écaille, pour accentuer l'effet du visage, rendant ainsi évidente l'utilisation du relief.

51 Fort heureusement, le moulage nous a permis de retrouver le tracé d'origine. Nous avons donc effectué un nouveau relevé, à partir duquel nous proposons une autre lecture de l'anthropomorphe. D'abord, comme déjà noté par B. et G. Delluc (1987), celui-ci n'a pas de "chevelure "; les tracés situés derrière la tête sont plus fins et appartiennent sans doute à une autre représentation, que nous nous emploierons à étudier prochainement. 
Figure 11 - Tête 25, représentation gynoïde 26 et cheval 27. a. Relevé analytique (d'après Delluc et al. 1987). b. Relevé Dams 1980. c. Photomontage H. Paitier. d. Relevé analytique : les pointillés noirs représentent des ébauches de tracés. En rouge, les tracés modernes. En pointillés gris, les limites des épanchements de gypse (relevé F. Berrouet)
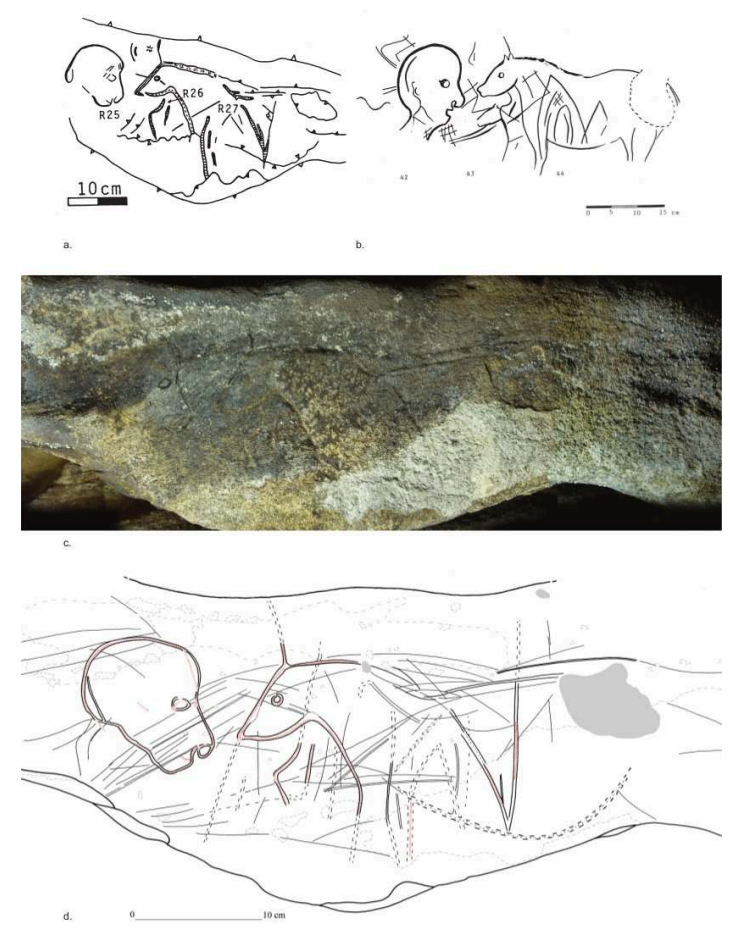

Figure 12 - Panneau du «Sorcier ». a. Premier relevé du " Sorcier » par André Glory (d'après Blanc 1955). b. Relevé par Dams 1980. c. Relevé analytique (d'après Delluc et al. 1987).

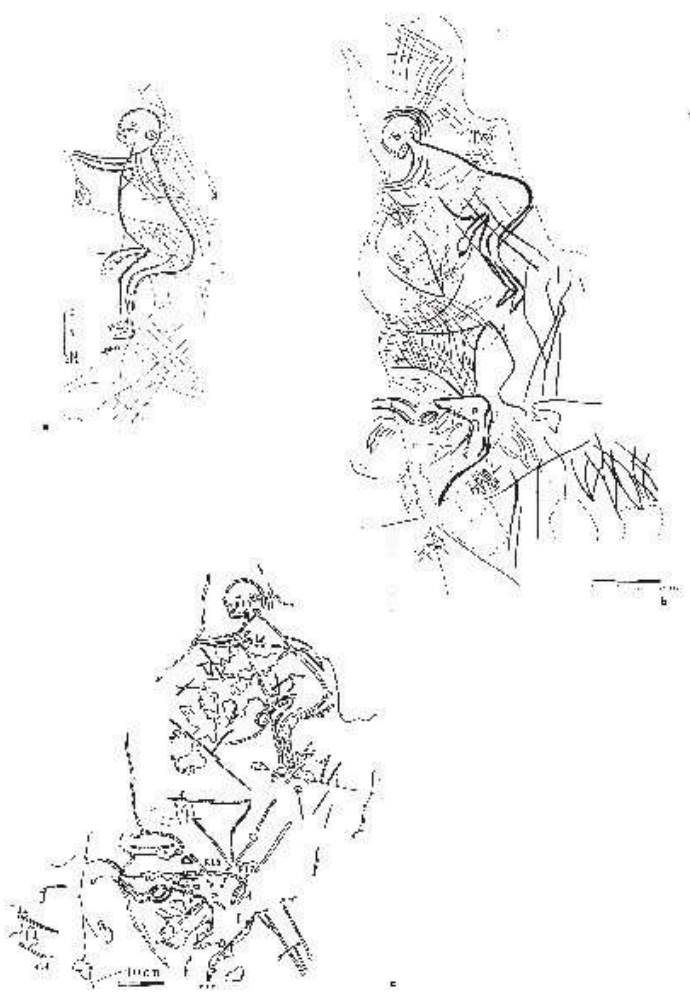


Figure 13 - a. Première languette reconstituée du panneau du « Sorcier » (photos et montage $\mathrm{H}$. Paitier). b. Relevé analytique du panneau du « Sorcier ». Les éléments de relief sont en grisé (relevé R. Pigeaud, avec la collaboration de F. Berrouet et E. Bougard).

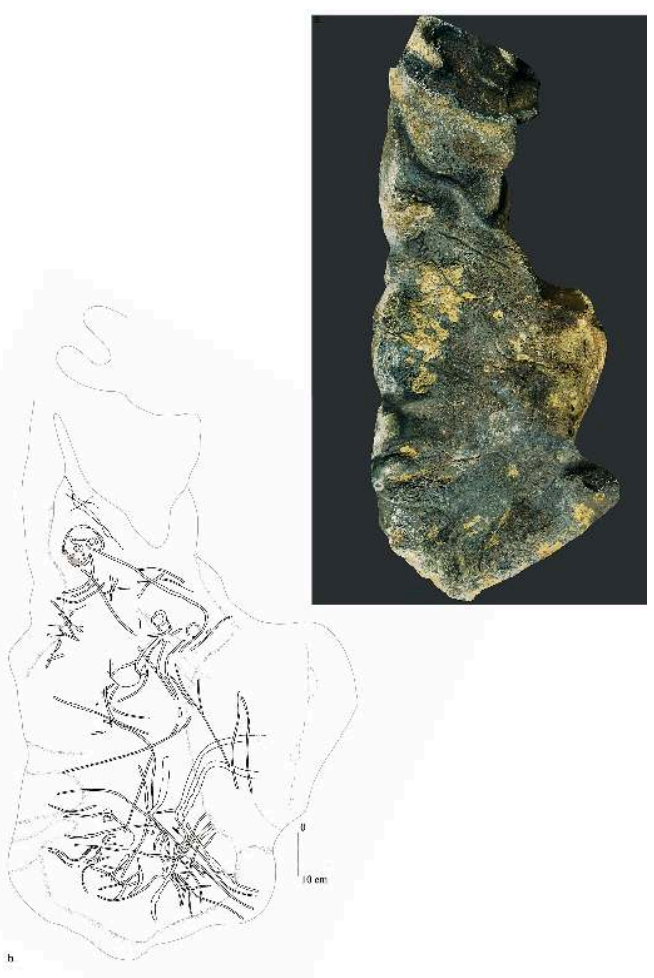


Figure 14 - Panneau du « Sorcier ». Relevé analytique. a. En jaune, les arrachements de paroi. b. En bleu, les limites des épanchements de gypse. c. Détériorations modernes : en rouge, les nouveaux tracés ; en brun, les traces de projection d'argile (coups de coude ou d'épaule) (relevé R. Pigeaud, avec la collaboration de F. Berrouet et E. Bougard).

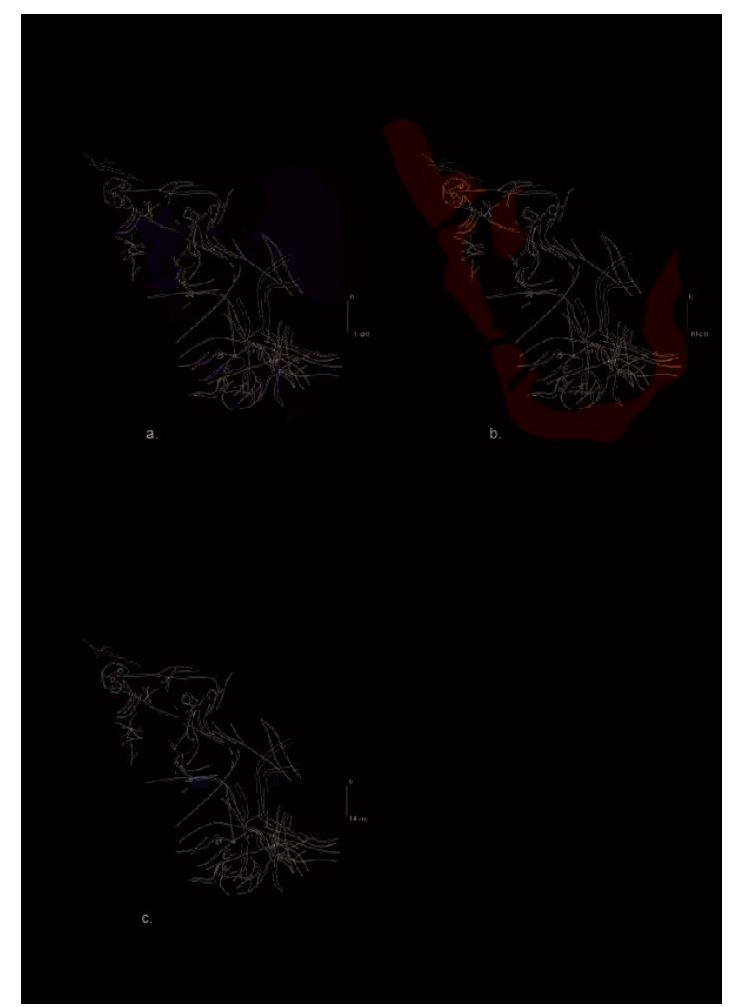

Pour ce qui est de la tête :

- trois tracés subsistent, au-dessus des yeux et entre ceux-ci ainsi qu'au-dessus de la mâchoire, qui soit appartiennent à une autre représentation, soit sont des traits de construction;

- il ne s'agit pas d'un œil et d'une oreille, mais bien de deux yeux, comme semblent le suggérer la similitude des tracés et leur volume équivalent; ce qui signifie, selon nous, que l'anthropomorphe a la tête figurée de face, comme beaucoup d'anthropomorphes évoqués en masques, « sorciers » et « fantômes » de l'art paléolithique ;

- la présence d'un tracé en avant de la tête vient confirmer cette hypothèse : il ferme le contour et recoupe la fameuse écaille dans laquelle tout le monde voit une utilisation naturelle pour figurer un profil. Selon nous, bien qu'elle semble avoir préexisté au tracé, cette écaille ne fut en rien sollicitée par l'artiste préhistorique. L'anthropomorphe a bien la tête de face. Ce qui d'ailleurs, là aussi, est cohérent avec l'ensemble du corpus connu, par exemple avec le fameux « Dieu cornu » de la grotte des Trois-Frères (Ariège).

53 Autre interprétation nouvelle: nous avons été longtemps intrigués par une forme arrondie tracée sur le bas du ventre de l'anthropomorphe. Un examen approfondi a démontré qu'il s'agissait d'un sabot bouleté, provenant de la jambe arrière gauche d'un cheval, dont l'autre jambe se situe un peu plus bas, en partie recouverte par le tracé de la fesse gauche du «Sorcier ». Partant de là, il a été possible de retrouver le tracé de cet équidé, figuré en profil gauche, en perspective bi-angulaire oblique, en posture dynamique (fig. 15). Aujourd'hui recouvert en partie par l'anthropomorphe et le bison, il apparaît déformé sur le relevé car il se situe dans un creux prononcé de la voûte. 
54 Grâce à l'étude des superpositions, nous avons pu reconstituer les étapes de la réalisation de l'anthropomorphe, aux dépens de l'équidé (fig. 15). En particulier, le phallus et la jambe gauche de l'anthropomorphe ont été tracés à partir du postérieur droit du cheval (fig. 16).

Cette découverte met en avant une véritable stratigraphie des tracés dans la grotte, en particulier dans ce secteur.

Figure 15 - Panneau du «Sorcier». a. b. c. d. Relevés synthétiques montrant les différentes étapes du tracé de l'anthropomorphe, établies à partir de l'étude des superpositions. e. Relevé synthétique montrant les tracés du cheval E (relevés R. Pigeaud, avec la collaboration de $F$. Berrouet et $E$. Bougard).

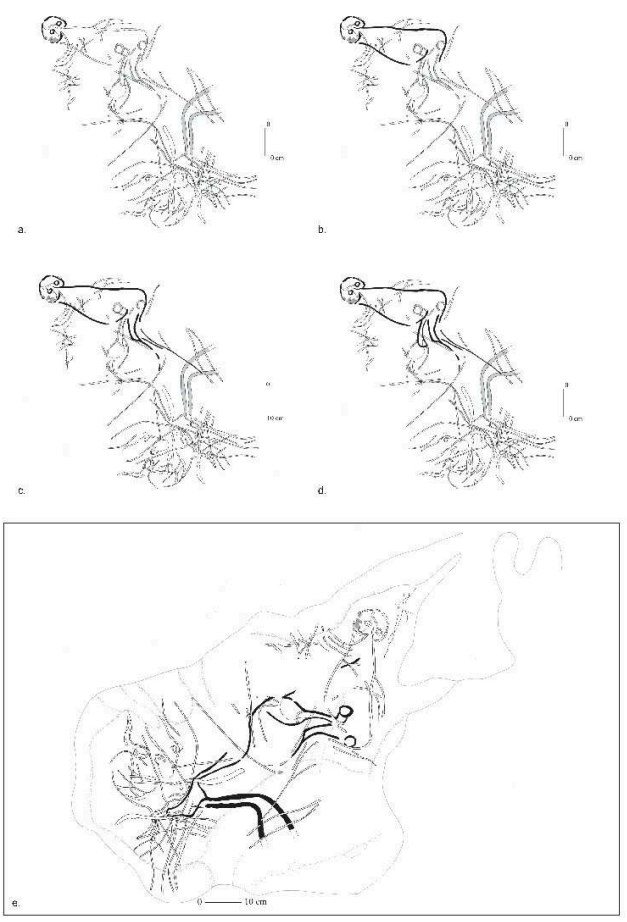


Figure 16 - Panneau du « Sorcier ». Détail du phallus montrant les deux sabots bouletés (photo H. Paitier).

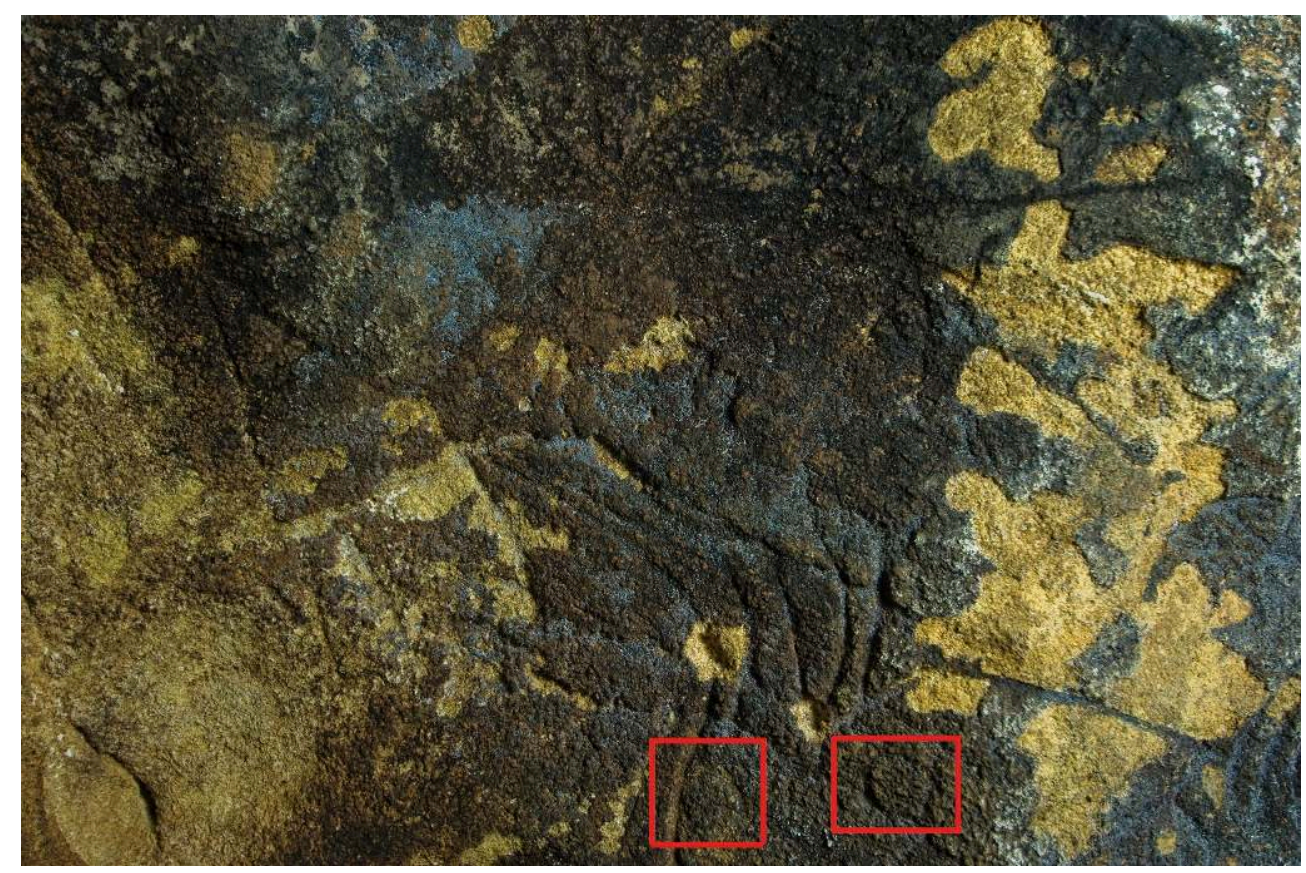

\section{Secteur VI, voûte : unité graphique $n^{\circ} 28$ (Secteur VI, voûte, panneau XIII)}

56 Au plafond de la salle, à l'aplomb du bord gauche de la tranchée en regardant vers le fond de la grotte, B. et $G$. Delluc avaient relevé quelques tracés qu'ils avaient décrits comme un " animal fragmentaire » (ibid, p. 388), réduit à son dos, le départ du poitrail et le possible contour allongé d'une cuisse (fig. 17). Ces traits anthropiques de 3 à $5 \mathrm{~mm}$ de large se trouvent sur une surface à peu près plane, très altérée, en partie desquamée au centre de la composition. Elle porte des vestiges d'un placage argileux ainsi que des nappages diffus de gypse (avec toutefois quelques efflorescences plus soutenues, certaines épaisses même de plusieurs millimètres) et est encadrée par des concavités naturelles; le fond des tracés est recouvert d'une pellicule argileuse noirâtre fendillée par la dessiccation. Par endroits, et notamment au niveau de bourrelets de calcaire affleurant en périphérie des coupoles de la voûte, des incisions plus profondes peuvent correspondre au fond de traits initialement plus larges et dont les bords ont aujourd'hui disparu.

La lecture que nous avons faite de ces tracés, à la faveur de nouveaux éclairages et d'un plancher métallique autorisant une proximité immédiate avec le plafond, nous a permis de poursuivre le trait de poitrail décrit par B. et G. Delluc de part et d'autre de la surface desquamée, ce dernier rejoignant ainsi le bord d'une concavité d'ouverture subcirculaire; c'est le cas également de la ligne de cuisse. La courbure constituant la jonction entre la ligne de dos et la ligne de cuisse, quoique nettement altérée et atténuée, existe bien cependant, d'après notre interprétation. À ce niveau, plusieurs traits possiblement gravés sont visibles, soit pour marquer la courbure, soit parce que d'autres représentations figuratives ou non, qu'il ne nous a pas été possible de déchiffrer pour le moment, recoupent les tracés et se développent dans les concavités. 
Un segment long d'environ $14 \mathrm{~cm}$ est perpendiculaire à la ligne de dos décrite par B. et G. Delluc, qu'il recoupe légèrement. La présence de nombreuses cupules coalescentes, formant des alignements qu'une lecture trop rapide inciterait à prendre pour des tracés, suppose la plus grande prudence dans la description des traits.

Cette composition (55 $\mathrm{cm}$ de plus grande dimension) nous évoque plutôt une représentation féminine schématique (FFS : Delluc et Delluc 1995) en profil gauche, en faisant pivoter le sens de lecture initialement proposé de $90^{\circ}$ dans le sens inverse des aiguilles d'une montre : le tracé courbe constituant ainsi la ligne de dos, la fesse et le départ de la jambe, tandis qu'un tracé subrectiligne suggère la partie antérieure, légèrement infléchi au niveau de la jonction entre la ligne de torse et le haut de la jambe. L'une des concavités vers laquelle convergent les deux tracés principaux pourrait avoir été intégrée comme forme naturelle de relief (tête?) par l'artiste paléolithique - nous n'y avons cependant pas relevé de tracés anthropiques.

Figure 17 - Représentation 28, interprétée auparavant comme une figuration animale. a. Relevé analytique (d'après Delluc et al. 1987), publié avec le numéro d'inventaire 32. b. Relevé Dams 1980 initialement publié à l'envers. c. Photo prise sous un autre angle : une autre interprétation est alors possible (photo $\mathrm{H}$. Paitier). d. Représentation 28 , interprétée comme une figure féminine schématique, dont la tête peut être figurée par une concavité. Les pointillés rouges indiquent les limites des épanchements de gypse, les pointillés noirs, les tracés probables (relevé $F$. Berrouet et E. Bougard).
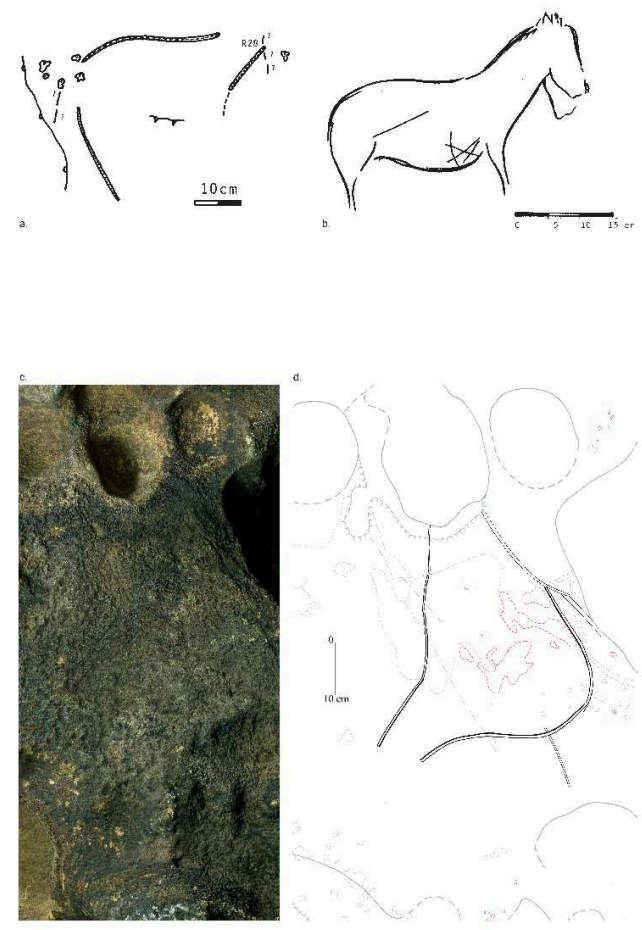

\section{Conclusion}

Reprendre l'étude d'une grotte ornée à la suite de préhistoriens réputés pour leur professionnalisme et le sérieux de leurs travaux constitue un programme ambitieux, au long duquel il importe de rester humble tout en étant objectif et attentif au moindre tracé susceptible de conduire à une nouvelle lecture d'une figure. D'une manière 
générale, nous n'avons pas rencontré d'objections majeures aux descriptions revues et corrigées que nous avons proposées: d'une part, parce qu'un certain nombre de modifications de traits sont à mettre au compte des actes de vandalisme remarqués sur certains panneaux gravés; d'autre part, parce qu'un consensus est généralement de mise face à une paroi éclairée différemment, ou même simplement vue depuis un angle inédit (les passerelles installées parallèlement à la voûte nous ont permis d'approcher les gravures comme jamais auparavant). Les discussions se sont cristallisées autour de la figure du "Sorcier ", représentation anthropomorphe emblématique de la grotte, pour laquelle non seulement nous proposons une nouvelle interprétation du tracé de la tête, mais encore nous y adjoignons, en vis-à-vis et renversée, une représentation d'équidé. Nous sommes prêts à soumettre notre lecture à nos collègues, dont certains nous ont déjà poussés dans nos retranchements. De nouvelles prises de vues, ainsi que le relevé des secteurs non accessibles pour le moment, permettront, nous l'espérons, de clore la discussion.

Notre connaissance de la grotte du Sorcier progresse donc, facilitée par l'établissement d'une topographie précise des lieux permettant la localisation dans l'espace des représentations et des altérations subies par la paroi, anthropiques et naturelles. L'étude des enlèvements de matière autorise des discussions avancées sur la position du remplissage avant la réouverture et l'aménagement de la cavité à l'époque moderne (voir Delluc et al. 1987 pour la chronologie). Quant à la découverte de gravures encore inédites ou aux précisions apportées à certains détails de relevés obtenus par nos prédécesseurs, elles montrent que le potentiel graphique n'est pas épuisé. Trois séquences sont perceptibles :

1. des reliefs appuyés proches du bas-relief;

2. des gravures prononcées, comme sur le panneau du «Sorcier», qui a vu au moins deux étapes se succéder; et

3. des gravures plus fines. Une chronologie relative qui demande évidemment à être affinée...

61 Près de cinquante ans après la découverte de la grotte du Sorcier, et vingt-cinq ans après la dernière étude systématique sérieuse des gravures par B. et G. Delluc, porter un regard neuf sur cet art pariétal du Périgord s'avère un choix non dénué d'intérêts, certains majeurs, eu égard à la compréhension de la paroi ornée que la démarche préhistorienne s'efforce de mettre en place aujourd'hui. Cette étape constitue l'aboutissement des travaux pionniers qui se sont succédé en ces lieux. Les explorations spéléologiques de Bernard Mortureux et le regard aiguisé de Séverin Blanc et Henri Breuil, au mitan du siècle dernier, ont permis les premières découvertes; les travaux de décalque des gravures menés par André Glory, accompagnés de moulages partiels des panneaux en 1966, ont précisé de nombreux détails et constitué les prémices du souci de conservation des parois (même si l'opération a laissé des traces) ; enfin, les relevés quasi exhaustifs exécutés par B. et G. Delluc en 1982 ont permis l'attribution chronologique des gravures, tandis que l'on percevait peu à peu l'organisation en composition articulée autour d'une représentation masculine complétée d'animaux ou de segments animaux, ainsi que de tracés géométriques. Mais les matériels et procédés mis à disposition des préhistoriens évoluent rapidement, la prise de vues photographiques se perfectionne, tandis que le recours à des éclairages en lumière froide autorise une communion exceptionnelle du regard avec les microreliefs pariétaux. Dans le même temps, en laboratoire, il est possible de réaliser des assemblages photographiques, de s'éloigner instantanément d'un détail de paroi pour 
embrasser d'un seul coup d'œil le panneau tout entier, de faire pivoter les figures pour en mieux cerner les intrications, les correspondances. Qui plus est, les dégradations malheureuses que nous avons amèrement pu constater sur certaines gravures, probablement commises peu avant la disparition de l'ancien propriétaire Ernest Paluzanno et le rachat de la grotte par le propriétaire actuel, au cours d'une période de « flottement » dans la vigilance apportée à la préservation du site, témoignent plus que jamais, s'il en était besoin, de l'extrême fragilité d'un environnement souterrain qui a su braver les siècles mais qu'il importe en permanence de protéger.

Les auteurs tiennent à saluer les collègues et spécialistes venus leur rendre visite au cours de ces campagnes de novembre-décembre 2010 et 2011 : J. Airvaux, J. Audoin, Norbert Aujoulat ( $\dagger$ ), D. Barraud, G. Bosinski, J.-J. Cleyet-Merle, C. Cretin, É. Crubézy, B. et G. Delluc, N. Fourment, D. Giovannacci, E. Man-Estier, M. Maumont, P. Paillet, S. Petrognani, J.-Ch. Portais, P. Raux, É. Robert, G. Sauvet, D. Tauxe et S. Touron. Que J.-M. Touron, propriétaire de la grotte du Sorcier et du gite associé dont nous avons fait notre laboratoire de terrain, trouve ici l'expression de nos plus sincères remerciements.

\section{BIBLIOGRAPHIE}

AUJOULAT N. 2006 - Art pariétal et milieu naturel. Mémoire d'Habilitation à diriger des recherches soutenu le 19 avril 2006 à l'Université Bordeaux 1.

BLANC S. 1955 - Une nouvelle grotte ornée près des Eyzies. La grotte Noël Brousse à Saint-Cirq. In : Collectif, Congrès préhistorique de France, compte rendu de la XIVe session, Strasbourg-Metz 1953, éditions de la SPF, p. 178-182.

BOSINSKI G. 2011 (avec la collaboration de É. BRUNEL, J.-M. CHAUVET et R. PIGEAUD) - Femmes sans tête. Une icône culturelle dans l'art européen de la fin de l'époque glaciaire. Paris : Errance, $232 \mathrm{p}$.

BOURDIER C. 2009-2010 - Histoires de bisons et de chevaux : regard sur l'évolution de la frise pariétale de Cap-Blanc (Marquay, Dordogne) à travers l'analyse du panneau de l'alcôve. Paleo, $\mathrm{n}^{\circ} 21, \mathrm{p} .17-38$.

BRUNET J., VIDAL P. 1978 - Grotte Sous-le-Roc de Saint-Cirq-du-Bugue (Dordogne). Étude des conditions climatiques. Intervention. Conservation. Spéléo-Dordogne, $\mathrm{n}^{\circ} 66$, p. 2-10.

CLOTTES J. 1998 - Voyage en Préhistoire. L'art des cavernes et des abris, de la découverte à l'interprétation. Paris : éd. La maison des roches, $479 \mathrm{p}$.

CLOTTES J., GARCIA M.-A., GUICHARNAUD R., LAUTIER J., LORBLANCHET M., ROUZAUD F., VIALOU A. et D. 1981 - Vrais et faux bisons de Mayrière-Supérieure (Bruniquel, Tarn-etGaronne) : problèmes d'observation et de méthode. BSPF, t. 78, n 3, p. 71-74 (rééd. Clottes 1998, p. 66-71).

COULSON D., CAMPBELL A., CLOTTES J. 2011 - Pas vraiment des faux, mais... International Newsletter On Rock Art, $\mathrm{n}^{\circ}$ 61, p. 26-29.

DAMS L. 1980 - L'art pariétal de la grotte du Roc Saint-Cirq. BAR International Series, 79, Oxford, $149 \mathrm{p}$.

PALEO, 23 | 2012 
DAMS L. et DAMS M. 1979 - La grotte de Mayrière-Supérieure à Bruniquel (Tarn-et-Garonne). Bull. Soc. Roy. Belge Anthrop. Préhist., 90, p. 85-98.

DELLUC B. et DELLUC G. 1984 - Grotte de Saint-Cirq. In : Collectif, L'Art des Cavernes. Atlas des grottes ornées paléolithiques françaises, Ministère de la Culture, Imprimerie Nationale, p. 210-213.

DELLUC B. et DELLUC G. 1995 - Les figures féminines schématiques du Périgord. L'Anthropologie, 99, p. 236-257.

DELLUC B. et DELLUC G. 2009 - Les découvertes d'art pariétal en Dordogne depuis un demi-siècle (1947-2000). Spelunca, mémoires n 34, p. 33-64.

DELLUC B., DELLUC G., GUICHARD F. 1987 - La grotte ornée de Saint-Cirq (Dordogne). BSPF, t. 84, $\mathrm{n}^{\circ}$ 10-12, Hommage de la SPF à André Leroi-Gourhan, p. 364-393.

DUHARD J.-P. 1995 - Réalisme de l'image masculine paléolithique. Grenoble : éd. Jérôme Millon, 256 p.

GARCIA M.-A. 1989 - La sculpture pariétale préhistorique. Technologie et terminologie. In :

Collectif, La sculpture rupestre en France (de la Préhistoire à nos jours), Actes du colloque de Brantôme, 14 août 1988, Société Historique et Archéologique du Périgord, supplément au bulletin, t. CXVI, p. 17-24.

GLORY A. 1964 - L'énigme de l'art quaternaire peut-elle être résolue par la théorie du culte des Ongones ? Revue des Sciences religieuses, 4 (oct.), p. 337-388.

LE QUELLEC J.-L. 2004 - Notice « Galop volant ». In : Collectif (dir. D. Vialou), La Préhistoire. Histoire et dictionnaire, coll. « Bouquins », éd. Robert Laffont, p. 662.

MALAURENT Ph., BRUNET J., LACANETTE D., CALTAGIRONE J.-P. 2005 - Apport de la modélisation numérique à la compréhension de l'état des parois de grottes préhistoriques. Premiers résultats à Lascaux. Préhistoire, art et sociétés, t. LX, p. 53-59.

PIGEAUD R. 1999 - Autour du Cap-Blanc : quelques remarques sur la «forme-cheval ». L'Anthropologie, 103 (4), p. 569-616.

PIGEAUD R. 2004 (avec la collaboration de M. BOUCHARD et É. LAVAL) - La grotte ornée Mayenne-Sciences (Thorigné-en-Charnie, Mayenne) : un exemple d'art pariétal d'époque gravettienne en France septentrionale. Gallia Préhistoire, 46, p. 1-154.

REINACH S. 1900 - La représentation du galop dans l'art ancien et moderne (première partie). Revue Archéologique, IIIème série, t. XXXVI, janvier-juin, p. 216-251, 46 fig., 1 tabl.

SAUVET G. 1988 - La communication graphique paléolithique (de l'analyse quantitative d'un corpus de données à son interprétation sémiologique). L'Anthropologie, t. 92, n 1, p. 3-15.

TOSELLO G. 1995 - Traces et simulacre en art préhistorique : éloge et critique de la reconstitution. Cahier Art et Science, nº 2, Publication de l'Université Bordeaux 1, p. 83-95.

TYMULA S. 2002 - L'art solutréen du Roc de Sers (Charente). Documents d'Archéologie Française, 91, Ministère de la Culture et de la Communication, Ministère de la Recherche, CNRS, Paris : éd. de la Maison des Sciences de l'Homme, $288 \mathrm{p}$.

\section{NOTES}

1. On a souvent reproché à cet auteur son manque de rigueur et sa propension à gonfler inconsidérément les inventaires (Clottes et al. 1981; Clottes 1998 ; Dams et Dams 1979), ce que nous avons pu vérifier dans la grotte Mayenne-Sciences (Mayenne), les grottes de Nerja et La 
Pileta (Andalousie, Espagne) et la grotte du Sorcier. L. Dams interprète trop souvent les accidents naturels et les confond avec des tracés paléolithiques. Pour autant, nous avons choisi, contrairement à B. et G. Delluc (1987 - note 6, p. 393), de prendre en compte ses relevés, justement parce qu'elle relève tout sans trier; ils constituent ainsi un bon outil de travail pour effectuer des repérages, avant le tri final.

2. Site Internet : www.grottedusorcier.com

3. Nous revendiquons ce terme, déjà employé à plusieurs reprises précédemment, car intervenir sur une paroi classée Monument historique et inscrite sur la liste du patrimoine mondial de l'humanité est un acte délictueux, quelles que soient les intentions initiales de la personne. Voir à ce sujet Coulson et al. 2011.

4. Nous rappelons qu'un panneau, suivant la définition de G. Sauvet $(1988$, p. 5), est «un ensemble plastique (peinture, gravure, sculpture, modelage) identifiable par ses limites physiques ». Celles-ci sont les fissures ou les limites des reliefs.

5. Les figures numérotées de A à J sont des figures complémentaires, proposées uniquement dans la version en ligne de l'article.

6. Relief semi-méplat selon la terminologie de S. Tymula (Tymula 2002).

7. Cette expression historique est employée dans le cas de chevaux «représentés les [jambes] raides, étendues parallèlement deux à deux vers l'avant et vers l'arrière, ce qui donne l'impression que l'animal ne touche pas le sol (bien que celui-ci ne soit jamais figuré)» (Le Quellec 2004). Une illusion d'optique, donc, en quelque sorte, devenue un terme descriptif propre aux préhistoriens et dont S. Reinach (1900) a assis la définition: le cheval est ainsi « complètement détaché du sol [...], les sabots sont tournés vers le dehors, leur base étant plus ou moins verticale à la ligne de terre, au lieu de se confondre avec elle »; le cheval paraît ainsi «très près du sol, au lieu d'en être plus éloigné qu'aux autres allures ».

8. Le calcaire coniacien est facile à travailler une fois débarrassé de son calcin (Tosello 1995 ; Pigeaud 1999).

\section{RÉSUMÉS}

Les progrès réalisés ces dernières années dans les moyens mis à disposition pour étudier l'art pariétal préhistorique, ainsi que l'amer constat d'actes de vandalisme opérés sur certaines gravures de la grotte du Sorcier à Saint-Cirq-du-Bugue (Dordogne), nous ont conduits à reprendre l'étude de ce site majeur de l'art aquitain des populations de chasseurs-collecteurs. Cette nouvelle campagne de relevés, ainsi que l'attachement à comprendre l'exécution des gravures en lien avec les formes de relief souterrain, nous permettent de mieux appréhender l'environnement physique de la grotte au moment de la réalisation des œuvres, tout en nous faisant une idée plus précise des gestes et des techniques employés. Par ailleurs, nous avons pu réaliser lors de la première campagne, menée à l'automne 2010, la couverture topographique complète de la cavité associée à la cotation précise de l'ensemble des représentations, en vue de la réalisation future d'un modèle numérique de terrain.

Sur la plupart des gravures réexaminées jusqu'alors, il nous a été possible de préciser certains tracés anciens, d'identifier la position des ajouts modernes et de constater les effets des altéragènes, associés à un support rocheux par endroits particulièrement vulnérable. La figure maîtresse de la grotte, cette représentation humaine ithyphallique, a fait l'objet d'une nouvelle lecture (notamment de la tête), qui a mis en évidence la gravure d'un équidé dont l'une des 
jambes arrière se superpose pour partie avec le tracé du sexe du "Sorcier». Une nouvelle gravure figurant un équidé, jusque-là passée inaperçue, a également été découverte. Enfin, au fur et à mesure de nos investigations, il nous est apparu essentiel d'étudier l'ensemble des gravures comme appartenant à une composition se développant autour d'une large fissure présente à la voûte - signe de l'importance des formes naturelles des parois dans l'articulation des représentations -, ainsi que de repenser la constitution de son décor comme la superposition de tracés de différentes époques.

Recent-years progress in the means available for the study of prehistoric parietal art as well as the bitter acknowledgement of vandalism on some engravings of the Sorcerer's cave in SaintCirq-du-Bugue (Dordogne) led us to start again the study of this major site for the art of the hunters-gatherers of Aquitaine. The new campaign of tracings, as well as the attention given to understanding the making of the engravings in connection with the underground relief shapes allowed a better apprehension of the artists' environment at the time of the making of the works of art, together with a more precise idea of the gestures and techniques used. Furthermore, during our first campaign in the autumn 2010, we were able to carry out a full topographic coverage of the cave associated with a precise sizing of all the engravings, in view of the future making of a numeric site model. On most of the studied engravings up to date, it was possible to precise some of the ancient lines, to identify the position of the modern lines and to record the effects of the degradation agents associated to a rock support that is especially fragile in places. The main figure of the cave, the human ithyphallic figure, was read anew (especially its head) which allowed us underlining the engraving of an equine whose back leg is partly superimposed to the engraving of the Sorcerer's sex. The new engraving of a horse, up to date unnoticed, was also discovered. Finally, in the course of our investigations, it appeared essential to us to study the whole of the engravings as belonging to one composition stretched around a large crack on the vault - showing how important the natural shapes of the rock surfaces are in the organisation of the representations - as well as to rethink the making of the decoration as the superimposition of lines from different time periods.

\section{INDEX}

Keywords : Sorcerer's cave, cave art, engravings, anthropomorph

Mots-clés : grotte du Sorcier, art pariétal, gravures, anthropomorphe

\section{AUTEURS}

\section{ROMAIN PIGEAUD}

Auteur correspondant. Responsable d'opération - UMR 7194 du CNRS, département de préhistoire du Muséum national d'Histoire naturelle, Institut de Paléontologie Humaine, 1 rue René Panhard, 75013 Paris - romain.pigeaud@wanadoo.fr

\section{FLORIAN BERROUET}

29 rue Dongaitz anaiak, 64122 Urrugne - florian.berrouet@gmail.com

\section{ESTELLE BOUGARD}

Le Maillet, 24580 Fleurac - ebougard@live.com 


\section{HERVÉ PAITIER}

Inrap Grand Ouest, 37 rue du Bignon, CS 67737, 35577 Cesson-Sévigné cedex - hervepierre.paitier@inrap.fr

\section{VINCENT POMMIER}

Inrap Grand Ouest, 37 rue du Bignon, CS 67737, 35577 Cesson-Sévigné cedex vincent.pommier@inrap.fr

\section{PASCAL BONIC}

Équipe spéléologique de l'ouest - martineetpascal@orange.fr 